\title{
Constant-Rank Codes and Their Connection to Constant-Dimension Codes
}

\author{
Maximilien Gadouleau and Zhiyuan Yan \\ Department of Electrical and Computer Engineering \\ Lehigh University, PA 18015, USA \\ E-mails: \{magc, yan $\} @$ lehigh.edu
}

\begin{abstract}
Constant-dimension codes have recently received attention due to their significance to error control in noncoherent random network coding. What the maximal cardinality of any constant-dimension code with finite dimension and minimum distance is and how to construct the optimal constant-dimension code (or codes) that achieves the maximal cardinality both remain open research problems. In this paper, we introduce a new approach to solving these two problems. We first establish a connection between constantrank codes and constant-dimension codes. Via this connection, we show that optimal constant-dimension codes correspond to optimal constant-rank codes over sufficiently large extension fields. As such, the two aforementioned problems are equivalent to determining the maximum cardinality of constant-rank codes and to constructing optimal constant-rank codes, respectively. To this end, we derive bounds on the maximum cardinality of a constant-rank code with a given minimum rank distance, propose explicit constructions of optimal or asymptotically optimal constant-rank codes, and establish asymptotic bounds on the maximum rate of a constant-rank code.
\end{abstract}

\section{INTRODUCTION}

While random network coding [1]-[3] has proved to be a powerful tool for disseminating information in networks, it is highly susceptible to errors caused by various sources such as noise, malicious or malfunctioning nodes, or insufficient min-cut. If received packets are linearly combined at random to deduce the transmitted message, even a single error in one erroneous packet could render the entire transmission useless. Thus, error control for random network coding is critical and has received growing attention recently. Error control schemes proposed for random network coding assume two types of transmission models: some [4]-[8] depend on and take advantage of the underlying network topology 
or the particular linear network coding operations performed at various network nodes; others [9], [10] assume that the transmitter and receiver have no knowledge of such channel transfer characteristics. The contrast is similar to that between coherent and noncoherent communication systems.

Error control for noncoherent random network coding was first considered in [9]1. Motivated by the property that random network coding is vector-space preserving, an operator channel that captures the essence of the noncoherent transmission model was defined in [9]. Similar to codes defined in complex Grassmannians for noncoherent multiple-antenna channels, codes defined in Grassmannians over a finite field [12], [13] and used with the subspace distance (cf. [9, (3)]) play a significant role in error control for noncoherent random network coding; Under the subspace distance, the weight of a subspace is simply its dimension; thus, we refer to these codes as constant-dimension codes (CDCs) henceforth. The standard advocated approach to random network coding (see, e.g., [2]) involves transmission of packet headers used to record the particular linear combination of the components of the message present in each received packet. From coding theoretic perspective, the set of subspaces generated by the standard approach may be viewed as a suboptimal CDC with minimum subspace distance 2 in the Grassmannian, because the Grassmannian contains more spaces with minimum subspace distance 2 than those obtained by the standard approach [9]. Hence, studying random network coding from coding theoretic perspective results in better error control schemes.

General studies of subspace metric codes (also referred to as codes in projective space or projective geometry) started only recently (see, for example, [14], [15]). On the other hand, there is a steady stream of works that focuses on codes in the Grassmannian. For example, Delsarte [12] proved that the Grassmannian endowed with the subspace distance forms an association scheme, and derived its parameters. The nonexistence of perfect codes in the Grassmannian was proved in [13], [16]. In [17], it was shown that Steiner structures yield diameter-perfect codes in the Grassmannian; properties and constructions of these structures were studied in [18]; in [19], it was shown that Steiner structures result in optimal CDCs. Related work on certain intersecting families and on byte-correcting codes can be found in [20] and [21], respectively. An application of codes in the Grassmanian to linear authentication schemes was considered in [22]. In [9], a Singleton bound for CDCs and a family of codes that are nearly Singleton-bound achieving are proposed, and a recursive construction of CDCs which outperform the codes in [9] was given in [23]. Despite the asymptotic optimality of the Singleton bound and the codes proposed in [9], both are not optimal in finite cases: upper bounds tighter than the Singleton bound

\footnotetext{
${ }^{1}$ A related work [11] considers security issues in noncoherent random network coding.
} 
exist and can be achieved in some special cases [19]. It is yet to be determined the maximal cardinality of a CDC with finite dimension and minimum distance, and it is not clear how to construct the optimal code (or codes) that achieves the maximal cardinality.

In this paper, we introduce a novel approach to solving the two aforementioned problems. Namely, we aim to solve these problems via rank metric codes, in particular, constant-rank codes (CRCs), which are the counterparts in rank metric codes of constant Hamming weight codes. There are several reasons for our approach. First, it is difficult to answer the two questions based on CDCs directly since the projective space lacks a natural group structure [10]. Second, the rank metric is similar to the Hamming metric and hence familiar results from the Hamming space can be readily adapted. Furthermore, there are extensive works on rank metric codes in the literature. Finally, the rank metric has been shown relevant to error control for both noncoherent [10] and coherent [24] random network coding.

Based on our approach, this paper makes two main contributions. Our first main contribution is that we establish a connection between CRCs and CDCs. Via this connection, we show that optimal CDCs correspond to optimal CRCs over sufficiently large extension fields. This connection converts the aforementioned open research problems about CDCs into research problems about CRCs, thereby allowing us to use rich results in rank metric codes to tackle such problems. Since constant-rank codes have received little attention in the literature, our second main contribution is our investigation of the properties of CRCs. In particular, we derive upper and lower bounds on the maximum cardinality of a CRC, propose explicit constructions of optimal or asymptotically optimal CRCs, and establish asymptotic bounds on the maximum rate of CRCs.

The rest of the paper is organized as follows. Section $\amalg$ reviews some necessary background. In Section III, we determine the connection between optimal CRCs and optimal CDCs. In Section IV] we study the maximum cardinality of CRCs, and we present our results on the asymptotic behavior of the maximum rate of a CRC.

\section{Preliminaries}

\section{A. Rank metric codes and elementary linear subspaces}

Error correction codes with the rank metric [25]-[27] have been receiving steady attention in the literature due to their applications in storage systems [27], public-key cryptosystems [28], space-time coding [29], and network coding [9], [10]. Below we review some important properties of rank metric codes established in [25]-[27]. 
Consider a vector $\mathbf{x}$ of length $n$ over $\operatorname{GF}\left(q^{m}\right)$. The field $\operatorname{GF}\left(q^{m}\right)$ may be viewed as an $m$-dimensional vector space over $\operatorname{GF}(q)$. The rank weight of $\mathbf{x}$, denoted as $\operatorname{rk}(\mathbf{x})$, is defined to be the maximum number of coordinates of $\mathbf{x}$ that are linearly independent over $\mathrm{GF}(q)$ [26]. For any basis $B_{m}$ of $\mathrm{GF}\left(q^{m}\right)$ over $\mathrm{GF}(q)$, each coordinate of $\mathrm{x}$ can be expanded to an $m$-dimensional column vector over $\mathrm{GF}(q)$ with respect to $B_{m}$. Thus the rank weight of $\mathrm{x}$ is also given by the rank of the $m \times n$ matrix over $\operatorname{GF}(q)$ obtained by expanding all the coordinates of $\mathrm{x}$ [26]. We shall assume that the expansions are with respect to a given basis $B_{m}$ of $\operatorname{GF}\left(q^{m}\right)$ over $\operatorname{GF}(q)$ henceforth.

For all $\mathbf{x}, \mathbf{y} \in \operatorname{GF}\left(q^{m}\right)^{n}$, it is easily verified that $d_{\mathrm{R}}(\mathbf{x}, \mathbf{y}) \stackrel{\text { def }}{=} \operatorname{rk}(\mathbf{x}-\mathbf{y})$ is a metric over $\operatorname{GF}\left(q^{m}\right)^{n}$, referred to as the rank metric henceforth [26]. The minimum rank distance and rank diameter of a code $C$, denoted as $d_{\mathrm{R}}$ and $D_{\mathrm{R}}$, respectively, are simply the minimum and maximum rank distance over all possible pairs of distinct codewords.

It is shown in [25]-[27] that the minimum rank distance of a block code of length $n$ and cardinality $M$ over $\operatorname{GF}\left(q^{m}\right)$ satisfies $d_{\mathrm{R}} \leq n-\log _{q^{m}} M+1$. In this paper, we refer to this bound as the Singleton bound for rank metric codes and codes that attain the equality as maximum rank distance (MRD) codes. We refer to the subclass of linear MRD codes introduced in [30] as generalized Gabidulin codes.

We denote the number of vectors of rank $r(0 \leq r \leq \min \{m, n\})$ in $\operatorname{GF}\left(q^{m}\right)^{n}$ as $N_{r}\left(q^{m}, n\right)=$ $\left[\begin{array}{l}n \\ r\end{array}\right] \alpha(m, r)$ [26], where $\alpha(m, 0) \stackrel{\text { def }}{=} 1, \alpha(m, r) \stackrel{\text { def }}{=} \prod_{i=0}^{r-1}\left(q^{m}-q^{i}\right)$, and $\left[\begin{array}{l}n \\ r\end{array}\right] \stackrel{\text { def }}{=} \alpha(n, r) / \alpha(r, r)$ for $r \geq 1$. The $\left[\begin{array}{l}n \\ r\end{array}\right]$ term is often referred to as a Gaussian polynomial [31]. The volume of a ball with rank radius $r$ in $\operatorname{GF}\left(q^{m}\right)^{n}$ is denoted as $V_{r}\left(q^{m}, n\right)=\sum_{i=0}^{r} N_{i}\left(q^{m}, n\right)$. We denote the intersection of two spheres in $\mathrm{GF}\left(q^{m}\right)^{n}$ of radii $r$ and $s$, with distance between their centers $d$ as $J\left(q^{m}, n, r, s, d\right)$. We will omit the dependence of the quantities defined above on $q^{m}$ and $n$ when there is no ambiguity about the vector space considered. By [32, Theorem 3.6], we obtain

$$
q^{m n} N_{d} J(r, s, d)=\sum_{l=0}^{n} N_{l} P_{r}(l) P_{s}(l) P_{d}(l),
$$

where $P_{j}(l)$ is a $q$-Krawtchouk polynomial [33], [34]:

$$
P_{j}(l)=\sum_{i=0}^{n}(-1)^{j-i} q^{i m+(j-i)(j-i-1) / 2}\left[\begin{array}{l}
n-l \\
n-j
\end{array}\right]\left[\begin{array}{c}
n-i \\
l
\end{array}\right] .
$$

For all $q, 1 \leq d \leq r \leq n \leq m$, the number of codewords of rank $r$ in an $(n, n-d+1, d)$ linear MRD code over $\operatorname{GF}\left(q^{m}\right)$ is given by [26]

$$
M\left(q^{m}, n, d, r\right) \stackrel{\text { def }}{=}\left[\begin{array}{l}
n \\
r
\end{array}\right] \sum_{j=d}^{r}(-1)^{r-j}\left[\begin{array}{l}
r \\
j
\end{array}\right] q^{(r-j)(r-j-1) / 2}\left(q^{m(j-d+1)}-1\right) .
$$

An elementary linear subspace (ELS) [35] is defined to be a linear subspace $\mathcal{V} \subseteq \operatorname{GF}\left(q^{m}\right)^{n}$ for which there exists a basis of vectors in $\operatorname{GF}(q)^{n}$. We denote the set of all ELSs of $\operatorname{GF}\left(q^{m}\right)^{n}$ with dimension 
$v$ as $E_{v}\left(q^{m}, n\right)$ and the set of all ELSs as $E\left(q^{m}, n\right)$. It can be easily shown that $\left|E_{v}\left(q^{m}, n\right)\right|=\left[\begin{array}{l}n \\ v\end{array}\right]$ for all $m$. An ELS has properties similar to those for a set of coordinates [35]. In particular, any vector belonging to an ELS with dimension $r$ has rank no more than $r$; conversely, any vector $\mathbf{x} \in \operatorname{GF}\left(q^{m}\right)^{n}$ with rank $r$ belongs to a unique ELS in $E_{r}\left(q^{m}, n\right)$.

\section{B. Subspace distance and constant-dimension codes}

For two subspaces of $\operatorname{GF}(q)^{n}, \mathcal{U}$ and $\mathcal{V}$, it is easily verified that

$$
d_{\mathrm{s}}(\mathcal{U}, \mathcal{V}) \stackrel{\text { def }}{=} \operatorname{dim}(\mathcal{U}+\mathcal{V})-\operatorname{dim}(\mathcal{U} \cap \mathcal{V})=2 \operatorname{dim}(\mathcal{U}+\mathcal{V})-\operatorname{dim}(\mathcal{U})-\operatorname{dim}(\mathcal{V})
$$

is a metric over $E(q, n)$, referred to as the subspace metric [9] henceforth. The subspace distance between $\mathcal{U}$ and $\mathcal{V}$ thus satisfies $d_{\mathrm{S}}(\mathcal{U}, \mathcal{V})=2 \operatorname{rk}\left(\mathbf{X}^{T} \mathbf{Y}^{T}\right)-\operatorname{rk}(\mathbf{X})-\operatorname{rk}(\mathbf{Y})$, where $\mathbf{X}$ and $\mathbf{Y}$ are generator matrices of $\mathcal{U}$ and $\mathcal{V}$, respectively. The minimum subspace distance and subspace diameter of any subspace metric code, denoted as $d_{\mathrm{S}}$ and $D_{\mathrm{S}}$, respectively, are the minimum and maximum subspace distance over all possible pairs of distinct subspaces.

A constant-dimension code $\Omega$ of length $n$ and constant-dimension $r$ over $\operatorname{GF}(q)$ is defined to be a nonempty subset of $E_{r}(q, n)$ [9]. When $\Omega$ has minimum subspace distance $d_{\mathrm{S}}$, we refer to $\Omega$ as an $\left(n, d_{\mathrm{S}}, r\right) \operatorname{CDC}$ over $\operatorname{GF}(q)$ and we denote the maximum cardinality of all $\left(n, d_{\mathrm{s}}, r\right) \operatorname{CDCs}$ over $\operatorname{GF}(q)$ as $A_{\mathrm{S}}\left(q, n, d_{\mathrm{S}}, r\right)$. Since $A_{\mathrm{S}}\left(q, n, d_{\mathrm{S}}, r\right)=A_{\mathrm{S}}\left(q, n, d_{\mathrm{S}}, n-r\right)$ [19], only the case where $2 r \leq n$ needs to be considered. By (4), it is easily verified that the subspace distance between any two subspaces of the same dimension is even; thus, the minimum distance of any CDC is even. Hence, we shall consider $A_{\mathrm{S}}(q, n, 2 d, r)$ for $2 \leq d \leq r$ henceforth since $A_{\mathrm{S}}(q, n, 2, r)=\left[\begin{array}{l}n \\ r\end{array}\right]$ and $A_{\mathrm{S}}(q, n, 2 d, r)=1$ for $d>r$. Upper and lower bounds on $A_{\mathrm{S}}(q, n, 2 d, r)$ were derived in [9], [14], [15], [19], [22]. In particular, for all $q, 2 r \leq n$, and $2 \leq d \leq r$, it was shown in [9], [22] that

$$
q^{(n-r)(r-d+1)} \leq A_{\mathrm{S}}(q, n, 2 d, r) \leq \frac{\alpha(n, r-d+1)}{\alpha(r, r-d+1)} .
$$

Note that the lower bound is both constructive and asymptotically tight [9], and we use this bound in our derivation of asymptotic rate of CRCs.

\section{Preliminary graph-theoretic results}

We review some results in graph theory given in [36]. We denote the set of vertices in a graph $G$ as $V(G)$, and the adjacency between any two vertices $u$ and $v$ as $u \sim v$.

Definition 1: Let $G$ and $H$ be two graphs. A mapping $f$ from $V(G)$ to $V(H)$ is a homomorphism if for all $u, v \in V(G), f(u) \sim f(v)$ if $u \sim v$. 
Definition 2: Let $G$ be a graph and $\phi$ a bijection from $V(G)$ to itself. $\phi$ is called an automorphism of $G$ if for all $u, v \in V(G), \phi(u) \sim \phi(v)$ if and only if $u \sim v$.

Definition 3: The graph $G$ is vertex transitive if for all $u, v \in V(G)$, there exists an automorphism $\phi$ of $G$ such that $\phi(u)=v$.

An independent set of a graph $G$ is a subset of $V(G)$ with no adjacent vertices. The independence number $\alpha(G)$ of $G$ is the maximum cardinality of an independent set of $G$. If $H$ is a vertex transitive graph and if there is a homomorphism from $G$ to $H$, then [36], [37]

$$
\alpha(G) \geq \alpha(H) \frac{|G|}{|H|} .
$$

\section{CONNECTION BETWEEN CONSTANT-DIMENSION CODES AND CONSTANT-RANK CODES}

In this section, we first establish some connections between the rank metric and the subspace metric. We then define constant-rank codes and we show how optimal constant-rank codes can be used to construct optimal CDCs.

For $\mathbf{x} \in \operatorname{GF}\left(q^{m}\right)^{n}$ and a basis $B_{m}$ of $\operatorname{GF}\left(q^{m}\right)$ over $\operatorname{GF}(q)$, let us consider $\mathbf{X} \in \operatorname{GF}(q)^{m \times n}$, the expansion of $\mathbf{x}$ with respect to $B_{m}$. Let us denote the row span and the column span of $\mathbf{X}$ over $\mathrm{GF}(q)$ as $\mathcal{R}\left(\mathbf{x} ; B_{m}\right)$ and $\mathcal{C}\left(\mathbf{x} ; B_{m}\right)$, respectively. Clearly, $\mathcal{C}\left(\mathbf{x} ; B_{m}\right) \in E_{r}(q, m)$ and $\mathcal{R}\left(\mathbf{x} ; B_{m}\right) \in E_{r}(q, n)$, where $r=\operatorname{rk}(\mathbf{x})$. We remark that $\mathcal{R}\left(\mathbf{x} ; B_{m}\right)$ does not depend on $B_{m}$ since changing a basis simply results in elementary row operations on $\mathbf{X}$; on the other hand, $\mathcal{C}\left(\mathbf{x} ; B_{m}\right)$ depends on $B_{m}$, even the order of the elements in $B_{m}$, although the dimension of $\mathcal{C}\left(\mathbf{x} ; B_{m}\right)$ is independent of $B_{m}$. We shall henceforth assume that the basis and the order of the basis elements are fixed and simply use the notations $\mathcal{R}(\mathbf{x})$ and $\mathcal{C}(\mathbf{x})$.

The notations introduced above are naturally extended to codes as follows: for $C \subseteq \operatorname{GF}\left(q^{m}\right)^{n}, \mathcal{C}(C) \stackrel{\text { def }}{=}$ $\{\mathcal{C}(\mathbf{c}): \mathbf{c} \in C\}$ and $\mathcal{R}(C) \stackrel{\text { def }}{=}\{\mathcal{R}(\mathbf{c}): \mathbf{c} \in C\}$.

Lemma 1: For $\mathcal{U} \in E_{r}(q, m), \mathcal{V} \in E_{r}(q, n)$, and $\mathbf{x} \in \operatorname{GF}\left(q^{m}\right)^{n}$ with rank $r, \mathcal{C}(\mathbf{x})=\mathcal{U}$ and $\mathcal{R}(\mathbf{x})=\mathcal{V}$ if and only if $\mathbf{X}=\mathbf{G}^{T} \mathbf{H}$, where $\mathbf{G} \in \mathrm{GF}(q)^{r \times m}$ is a generator matrix of $\mathcal{U}$ and $\mathbf{H} \in \mathrm{GF}(q)^{r \times n}$ is a generator matrix of $\mathcal{V}$.

The proof of Lemma 1 is straightforward and hence omitted. We remark that $\mathbf{X}=\mathbf{G}^{T} \mathbf{H}$ is referred to as a rank factorization [38]. Alternatively, $\mathbf{x}=\mathbf{b H}$, where the expansion (with respect to a basis of $\mathrm{GF}\left(q^{m}\right)$ over $\left.\mathrm{GF}(q)\right)$ of $\mathbf{b} \in \mathrm{GF}\left(q^{m}\right)^{r}$ is given by $\mathbf{G}^{T}$. We now derive a relation between the rank distance between two vectors and the subspace distances between their respective row and column spans.

Theorem 1: For all $\mathbf{x}, \mathbf{y} \in \operatorname{GF}\left(q^{m}\right)^{n}$, let us denote $d_{\mathrm{S}}(\mathcal{C}(\mathbf{x}), \mathcal{C}(\mathbf{y}))$ and $d_{\mathrm{S}}(\mathcal{R}(\mathbf{x}), \mathcal{R}(\mathbf{y}))$ as $d_{\mathcal{C}}$ and $d_{\mathcal{R}}$, respectively. Without loss of generality, assume $d_{\mathcal{C}} \geq d_{\mathcal{R}}$, then

$$
\frac{1}{2} \max \left\{d_{\mathcal{C}}+|\operatorname{rk}(\mathbf{x})-\operatorname{rk}(\mathbf{y})|, 2 d_{\mathcal{C}}+d_{\mathcal{R}}-\operatorname{rk}(\mathbf{x})-\operatorname{rk}(\mathbf{y})\right\} \leq d_{\mathrm{R}}(\mathbf{x}, \mathbf{y}) \leq \frac{1}{2}\left[d_{\mathcal{R}}+\operatorname{rk}(\mathbf{x})+\operatorname{rk}(\mathbf{y})\right] .
$$


Proof: We have $\mathcal{R}(\mathbf{x}-\mathbf{y}) \subseteq \mathcal{R}(\mathbf{x})+\mathcal{R}(\mathbf{y})$ and hence $d_{\mathrm{R}}(\mathbf{x}, \mathbf{y})=\operatorname{dim} \mathcal{R}(\mathbf{x}-\mathbf{y}) \leq \operatorname{dim}(\mathcal{R}(\mathbf{x})+$ $\mathcal{R}(\mathbf{y}))=\frac{1}{2}\left(d_{\mathcal{R}}+\operatorname{rk}(\mathbf{x})+\operatorname{rk}(\mathbf{y})\right)$ by (4).

Let $\mathbf{x}=\mathbf{b H}$ and $\mathbf{y}=\mathbf{b}^{\prime} \mathbf{H}^{\prime}$ so that $\mathbf{b} \in \mathrm{GF}\left(q^{m}\right)^{\mathrm{rk}(\mathbf{x})}$ and $\mathbf{b}^{\prime} \in \mathrm{GF}\left(q^{m}\right)^{\mathrm{rk}(\mathbf{y})}$. By definition of the subspace distance, $\operatorname{dim}(\mathcal{C}(\mathbf{x}) \cap \mathcal{C}(\mathbf{y}))=\frac{1}{2}\left[\operatorname{rk}(\mathbf{x})+\operatorname{rk}(\mathbf{y})-d_{\mathcal{C}}\right]$. Therefore, we can select $d_{1}=$ $\frac{1}{2}\left[\operatorname{rk}(\mathbf{x})-\operatorname{rk}(\mathbf{y})+d_{\mathcal{C}}\right]$ coordinates $\beta_{0}, \beta_{1}, \ldots, \beta_{d_{1}-1}$ of $\mathbf{b}$ linearly independent to $\mathbf{b}^{\prime}$, and $d_{2}=\frac{1}{2}[\operatorname{rk}(\mathbf{y})-$ $\left.\operatorname{rk}(\mathbf{x})+d_{\mathcal{C}}\right]$ coordinates $\beta_{0}^{\prime}, \beta_{1}^{\prime}, \ldots, \beta_{d_{2}-1}^{\prime}$ of $\mathbf{b}^{\prime}$ linearly independent to $\mathbf{b}$. By (4), it can be easily shown that $d_{\mathcal{C}} \geq|\operatorname{rk}(\mathbf{x})-\operatorname{rk}(\mathbf{y})|$. Let us select $\left\{\gamma_{d_{\mathcal{C}}}, \gamma_{d_{\mathcal{C}}+1}, \ldots, \gamma_{m-1}\right\}$ in $\operatorname{GF}\left(q^{m}\right)$ so that

$$
\gamma=\left\{\beta_{0}, \beta_{1}, \ldots, \beta_{d_{1}-1}, \beta_{0}^{\prime}, \beta_{1}^{\prime}, \ldots, \beta_{d_{2}-1}^{\prime}, \gamma_{d_{\mathcal{C}}}, \gamma_{d_{\mathcal{C}}+1}, \ldots, \gamma_{m-1}\right\}
$$

constitutes a basis of $\mathrm{GF}\left(q^{m}\right)$ over $\operatorname{GF}(q)$.

Expanding $\mathbf{x}-\mathbf{y}$ with respect to $\gamma$, we obtain $\operatorname{rk}(\mathbf{x}-\mathbf{y}) \geq \operatorname{rk}\left(\overline{\mathbf{H}}^{T} \overline{\mathbf{H}}^{\prime T}\right)$, where $\overline{\mathbf{H}}$ and $\overline{\mathbf{H}}^{\prime}$ are the $d_{1}$ rows of $\mathbf{H}$ and the $d_{2}$ rows of $\mathbf{H}^{\prime}$ corresponding to $\beta_{0}, \beta_{1}, \ldots, \beta_{d_{1}-1}$ and $\beta_{0}^{\prime}, \beta_{1}^{\prime}, \ldots, \beta_{d_{2}-1}^{\prime}$, respectively. First, we have $\operatorname{rk}\left(\overline{\mathbf{H}}^{T} \overline{\mathbf{H}}^{\prime T}\right) \geq \max \left\{\operatorname{rk}(\overline{\mathbf{H}}), \operatorname{rk}\left(\overline{\mathbf{H}}^{\prime}\right)\right\}=\max \left\{d_{1}, d_{2}\right\}$. Second, since $\left(\mathbf{H}^{T} \mathbf{H}^{\prime T}\right)$ has rank $\frac{1}{2}\left(d_{\mathcal{R}}+\operatorname{rk}(\mathbf{x})+\operatorname{rk}(\mathbf{y})\right)$ by (4), $\operatorname{rk}\left(\overline{\mathbf{H}}^{T} \overline{\mathbf{H}}^{\prime T}\right) \geq d_{\mathcal{C}}+\frac{1}{2}\left(d_{\mathcal{R}}-\operatorname{rk}(\mathbf{x})-\operatorname{rk}(\mathbf{y})\right)$.

Definition 4: A constant-rank code of length $n$ and constant-rank $r$ over $\operatorname{GF}\left(q^{m}\right)$ is a nonempty subset of $\operatorname{GF}\left(q^{m}\right)^{n}$ such that all elements have rank weight $r$.

We denote a CRC with length $n$, minimum rank distance $d$, and constant-rank $r$ as an $(n, d, r)$ CRC over $\operatorname{GF}\left(q^{m}\right)$. Proposition 1 below shows how a CRC leads to two CDCs with their minimum subspace distance and subspace diameter related to the minimum rank distance and rank diameter of the CRC.

Proposition 1: Let $C$ be an $\left(n, d_{\mathrm{R}}, r\right) \mathrm{CRC}$ over $\mathrm{GF}\left(q^{m}\right)$ with rank diameter $D_{\mathrm{R}}$. Then $\mathcal{R}(C) \subseteq$ $E_{r}(q, n)$ is a CDC with minimum subspace distance $d_{\mathcal{R}} \geq 2\left(d_{\mathrm{R}}-r\right)$. Similarly, $\mathcal{C}(C) \subseteq E_{r}(q, m)$ is a CDC with minimum subspace distance $d_{\mathcal{C}} \geq 2\left(d_{\mathrm{R}}-r\right)$. If we denote the subspace diameters of $\mathcal{R}(C)$ and $\mathcal{C}(C)$ as $D_{\mathcal{R}}$ and $D_{\mathcal{C}}$, respectively, then $\max \left\{D_{\mathcal{R}}, D_{\mathcal{C}}\right\} \leq \min \left\{2 D_{\mathrm{R}}, D_{\mathrm{R}}+r\right\}$.

Proof: By definition, $\mathcal{R}(C) \subseteq E_{r}(q, n)$ and $\mathcal{C}(C) \subseteq E_{r}(q, m)$. Let $\mathbf{x}$ and $\mathbf{y}$ be two distinct codewords in $C$ so that $d_{\mathrm{S}}(\mathcal{R}(\mathbf{x}), \mathcal{R}(\mathbf{y}))=d_{\mathcal{R}}$. By Theorem $1, d_{\mathcal{R}}=d_{\mathrm{S}}(\mathcal{R}(\mathbf{x}), \mathcal{R}(\mathbf{y})) \geq 2\left(d_{\mathrm{R}}(\mathbf{x}, \mathbf{y})-r\right) \geq$ $2\left(d_{\mathrm{R}}-r\right)$, and similarly $d_{\mathcal{C}} \geq 2\left(d_{\mathrm{R}}-r\right)$.

Let $\mathbf{x}_{0}$ and $\mathbf{y}_{0}$ be two codewords in $C$ such that $d_{\mathrm{s}}\left(\mathcal{C}\left(\mathbf{x}_{0}\right), \mathcal{C}\left(\mathbf{y}_{0}\right)\right)=D_{\mathcal{C}}$. By Theorem 1, we have $D_{\mathcal{C}} \leq 2 d_{\mathrm{R}}\left(\mathbf{x}_{0}, \mathbf{y}_{0}\right) \leq 2 D_{\mathrm{R}}$ and $2 D_{\mathcal{C}} \leq 2 d_{\mathrm{R}}\left(\mathbf{x}_{0}, \mathbf{y}_{0}\right)+2 r-d_{\mathrm{S}}\left(\mathcal{R}\left(\mathbf{x}_{0}\right), \mathcal{R}\left(\mathbf{y}_{0}\right)\right) \leq 2 D_{\mathrm{R}}+2 r$. Let $\mathbf{x}_{1}$ and $\mathbf{y}_{1}$ be two codewords in $C$ such that $d_{\mathrm{S}}\left(\mathcal{R}\left(\mathbf{x}_{1}\right), \mathcal{R}\left(\mathbf{y}_{1}\right)\right)=D_{\mathcal{R}}$, then by Theorem $1, D_{\mathcal{R}} \leq 2 D_{\mathrm{R}}$ and $2 D_{\mathcal{R}} \leq d_{\mathrm{R}}\left(\mathbf{x}_{0}, \mathbf{y}_{0}\right)+2 r-d_{\mathrm{S}}\left(\mathcal{C}\left(\mathbf{x}_{1}\right), \mathcal{C}\left(\mathbf{y}_{1}\right)\right) \leq 2 D_{\mathrm{R}}+2 r$.

When the minimum rank distance of a CRC is no less than its rank weight, Proposition 2 below shows how the CRC leads to two CDCs with the same cardinality, and the relations between their distances 
can be further strengthened.

Proposition 2: If $C$ is an $(n, d+r, r) \mathrm{CRC}$ over $\mathrm{GF}\left(q^{m}\right)(2 \leq d \leq r)$ with rank diameter $D_{\mathrm{R}}$, then $\mathcal{R}(C) \subseteq E_{r}(q, n)$ is a CDC with cardinality $|C|$ and minimum subspace distance $d_{\mathcal{R}} \geq 2 d$. Similarly, $\mathcal{C}(C) \subseteq E_{r}(q, m)$ is a CDC with cardinality $|C|$ and minimum subspace distance $d_{\mathcal{C}} \geq 2 d$. We also have $\frac{1}{2} \max \left\{2 d_{\mathcal{C}}+d_{\mathcal{R}}-2 r, 2 d_{\mathcal{R}}+d_{\mathcal{C}}-2 r\right\} \leq d+r$. If we denote the subspace diameters of $\mathcal{R}(C)$ and $\mathcal{C}(C)$ as $D_{\mathcal{R}}$ and $D_{\mathcal{C}}$, respectively, then $\max \left\{D_{\mathcal{R}}, D_{\mathcal{C}}\right\} \leq 2 D_{\mathrm{R}}, \min \left\{D_{\mathcal{R}}, D_{\mathcal{C}}\right\} \geq 2 D_{\mathrm{R}}-2 r$, and

$$
\frac{1}{2} \max \left\{D_{\mathcal{C}}+2 d_{\mathcal{R}}, D_{\mathcal{R}}+2 d_{\mathcal{C}}, 2 D_{\mathcal{C}}+d_{\mathcal{R}}, 2 D_{\mathcal{R}}+d_{\mathcal{C}}\right\} \leq D_{\mathrm{R}}+r
$$

Proof: Let $\mathbf{x}$ and $\mathbf{y}$ be any two distinct codewords in $C$. By Theorem $1, d_{\mathrm{S}}(\mathcal{R}(\mathbf{x}), \mathcal{R}(\mathbf{y})) \geq$ $2 d_{\mathrm{R}}(\mathbf{x}, \mathbf{y})-2 r \geq 2 d>0$, and hence $d_{\mathcal{R}} \geq 2 d$ and $|\mathcal{R}(C)|=|C|$. Similarly, $d_{\mathrm{s}}(\mathcal{C}(\mathbf{x}), \mathcal{C}(\mathbf{y})) \geq 2 d>0$, and thus $d_{\mathcal{C}} \geq 2 d$ and $|\mathcal{C}(C)|=|C|$. Furthermore, if $d_{\mathrm{R}}(\mathbf{x}, \mathbf{y})=d+r$, then by Theorem 11, $2(d+r) \geq$ $2 d_{\mathrm{S}}(\mathcal{C}(\mathbf{x}), \mathcal{C}(\mathbf{y}))+d_{\mathrm{s}}(\mathcal{R}(\mathbf{x}), \mathcal{R}(\mathbf{y}))-2 r \geq 2 d_{\mathcal{C}}+d_{\mathcal{R}}-2 r$. Similarly, we obtain $2(d+r) \geq 2 d_{\mathcal{R}}+d_{\mathcal{C}}-2 r$.

We now prove the inequalities involving $D_{\mathcal{C}}$. Let $\mathbf{x}_{0}$ and $\mathbf{y}_{0}$ be two codewords in $C$ such that $D_{\mathcal{C}}=d_{\mathrm{S}}\left(\mathcal{C}\left(\mathbf{x}_{0}\right), \mathcal{C}\left(\mathbf{y}_{0}\right)\right)$. By Theorem 1] we have $D_{\mathcal{C}} \leq 2 d_{\mathrm{R}}\left(\mathbf{x}_{0}, \mathbf{y}_{0}\right) \leq 2 D_{\mathrm{R}}, D_{\mathcal{C}} \leq 2 d_{\mathrm{R}}\left(\mathbf{x}_{0}, \mathbf{y}_{0}\right)+$ $2 r-2 d_{\mathrm{S}}\left(\mathcal{R}\left(\mathbf{x}_{0}\right), \mathcal{R}\left(\mathbf{y}_{0}\right)\right) \leq 2 D_{\mathrm{R}}+2 r-2 d_{\mathcal{R}}$, and $2 D_{\mathcal{C}} \leq 2 d_{\mathrm{R}}\left(\mathbf{x}_{0}, \mathbf{y}_{0}\right)+2 r-d_{\mathrm{S}}\left(\mathcal{R}\left(\mathbf{x}_{0}\right), \mathcal{R}\left(\mathbf{y}_{0}\right)\right) \leq$ $2 D_{\mathrm{R}}+2 r-d_{\mathcal{R}}$. Let $\mathbf{x}_{1}$ and $\mathbf{y}_{1}$ be two codewords in $C$ such that $d_{\mathrm{R}}\left(\mathbf{x}_{1}, \mathbf{y}_{1}\right)=D_{\mathrm{R}}$, then by Theorem 1 , $D_{\mathrm{R}} \leq \frac{1}{2}\left[d_{\mathrm{S}}\left(\mathcal{C}\left(\mathbf{x}_{1}\right), \mathcal{C}\left(\mathbf{y}_{1}\right)\right)+2 r\right] \leq \frac{1}{2}\left[D_{\mathcal{C}}+2 r\right]$. The other inequalities involving $D_{\mathcal{R}}$ are obtained similarly.

Using Lemma 1, we can construct CRCs in $\operatorname{GF}\left(q^{m}\right)^{n}$ from a pair of $\operatorname{CDCs}$ in $\operatorname{GF}(q)^{n}$ and $\operatorname{GF}(q)^{m}$, respectively.

Proposition 3: Let $\Gamma$ be an $\left(m, d_{\mathcal{C}}, r\right) \operatorname{CDC}$ over $\mathrm{GF}(q)$ and $\Delta$ be an $\left(n, d_{\mathcal{R}}, r\right) \operatorname{CDC}$ over $\mathrm{GF}(q)$. Then there exists a $\operatorname{CRC} C$ with length $n$, constant-rank $r$, and cardinality $\min \{|\Gamma|,|\Delta|\}$ over $\operatorname{GF}\left(q^{m}\right)$ satisfying $\mathcal{C}(C) \subseteq \Gamma$ and $\mathcal{R}(C) \subseteq \Delta$. Furthermore, its minimum distance $d_{\mathrm{R}}$ satisfies $\frac{1}{2} \max \left\{d_{\mathcal{C}}, d_{\mathcal{R}}\right\}+\frac{1}{2} \max \left\{d_{\mathcal{C}}+\right.$ $\left.d_{\mathcal{R}}-2 r, 0\right\} \leq d_{\mathrm{R}} \leq \frac{1}{2} \max \left\{d_{\mathcal{C}}, d_{\mathcal{R}}\right\}+r$. If we denote the subspace diameters of $\Delta$ and $\Gamma$ as $D_{\mathcal{R}}$ and $D_{\mathcal{C}}$, respectively, then the rank diameter $D_{\mathrm{R}}$ of $C$ satisfies $\frac{1}{2} \min \left\{D_{\mathcal{R}}, D_{\mathcal{C}}\right\} \leq D_{\mathrm{R}} \leq \frac{1}{2} \min \left\{D_{\mathcal{R}}, D_{\mathcal{C}}\right\}+r$ and $\min \left\{D_{\mathcal{C}}+2 d_{\mathcal{R}}, D_{\mathcal{R}}+2 d_{\mathcal{C}}, 2 D_{\mathcal{C}}+d_{\mathcal{R}}, 2 D_{\mathcal{R}}+d_{\mathcal{C}}\right\} \leq 2 D_{\mathrm{R}}+2 r$.

Proof: Denote the generator matrices of the component subspaces of $\Gamma$ and $\Delta$ as $\mathbf{G}_{i}$ and $\mathbf{H}_{i}$, respectively. Define the code $C$ formed by the codewords $\mathbf{c}_{i} \stackrel{\text { def }}{=} \mathbf{b}_{i} \mathbf{H}_{i}$ for $0 \leq i \leq \min \{|\Gamma|,|\Delta|\}-1$, where the expansion of $\mathbf{b}_{i} \in \mathrm{GF}\left(q^{m}\right)^{r}$ is given by $\mathbf{G}_{i}^{T}$. Then $\mathcal{C}(C) \subseteq \Gamma$ and $\mathcal{R}(C) \subseteq \Delta$ by Lemma 1 and the lower bound on $d_{\mathrm{R}}$ follows Theorem 1

By construction, $\mathcal{C}(C)=\Gamma$ or $\mathcal{R}(C)=\Delta$. If $\mathcal{C}(C)=\Gamma$, then let $\mathbf{x}$ and $\mathbf{y}$ be distinct codewords in $C$ such that $d_{\mathrm{S}}(\mathcal{C}(\mathbf{x}), \mathcal{C}(\mathbf{y}))=d_{\mathcal{C}}$. By Theorem 1, we obtain $d_{\mathrm{R}} \leq d_{\mathrm{R}}(\mathbf{x}, \mathbf{y}) \leq \frac{1}{2}\left(d_{\mathcal{C}}+2 r\right)$. Similarly, if 
$\mathcal{R}(C)=\Delta$, we obtain $d_{\mathrm{R}} \leq \frac{1}{2}\left(d_{\mathcal{R}}+2 r\right)$. Combining these results, we obtain $d_{\mathrm{R}} \leq \frac{1}{2} \max \left\{d_{\mathcal{R}}+2 r, d_{\mathcal{C}}+\right.$ $2 r\}$.

Let $\mathbf{x}$ and $\mathbf{y}$ be two codewords in $C$ such that $d_{\mathrm{R}}(\mathbf{x}, \mathbf{y})=D_{\mathrm{R}}$, then by Theorem $1,2 D_{\mathrm{R}} \leq d_{\mathrm{S}}(\mathcal{R}(\mathbf{x}), \mathcal{R}(\mathbf{y}))+$ $2 r \leq D_{\mathrm{S}}(\mathcal{R}(C))+2 r \leq D_{\mathcal{R}}+2 r$, and similarly $2 D_{\mathrm{R}} \leq D_{\mathcal{C}}+2 r$. If $\mathcal{C}(C)=\Gamma$, then let $\mathbf{x}^{\prime}$ and $\mathbf{y}^{\prime}$ be distinct codewords in $C$ such that $d_{\mathrm{S}}(\mathcal{C}(\mathbf{x}), \mathcal{C}(\mathbf{y}))=D_{\mathcal{C}}$; by Theorem $1, \frac{1}{2} D_{\mathcal{C}} \leq d_{\mathrm{R}}\left(\mathbf{x}^{\prime}, \mathbf{y}^{\prime}\right) \leq D_{\mathrm{R}}$. If $\mathcal{R}(C)=\Delta$, we obtain $\frac{1}{2} D_{\mathcal{R}} \leq D_{\mathrm{R}}$, and hence $\frac{1}{2} \min \left\{D_{\mathcal{C}}, D_{\mathcal{R}}\right\} \leq D_{\mathrm{R}}$. By a similar argument, we obtain $\min \left\{D_{\mathcal{C}}+2 d_{\mathcal{R}}, D_{\mathcal{R}}+2 d_{\mathcal{C}}, 2 D_{\mathcal{C}}+d_{\mathcal{R}}, 2 D_{\mathcal{R}}+d_{\mathcal{C}}\right\} \leq 2 D_{\mathrm{R}}+2 r$.

Proposition 4: Let $\Delta$ be an $(n, 2 d, r) \operatorname{CDC}$ over $\mathrm{GF}(q)$ with subspace diameter $D_{\mathrm{s}}$. Then for any extension field $\operatorname{GF}\left(q^{m}\right)$ with $m \geq n$, there exists a CRC $C$ of length $n$ and constant-rank $r$, over $\operatorname{GF}\left(q^{m}\right)$ such that $\mathcal{R}(C)=\Delta$ and $|C|=|\Delta|$. The minimum distance $d_{\mathrm{R}}$ of $C$ satisfies $2 d \leq d_{\mathrm{R}} \leq d+r$ and the rank diameter $D_{\mathrm{R}}$ of $C$ satisfies $\frac{1}{2} \max \left\{D_{\mathrm{S}}, 3 D_{\mathrm{S}}-2 r\right\} \leq D_{\mathrm{R}} \leq \frac{1}{2} D_{\mathrm{S}}+r$.

The proof of Proposition 4 follows Theorem 1, and is similar to that of Proposition 3 . The proof is given in Appendix A

We denote the maximum cardinality of an $(n, d, r) \operatorname{CRC}$ over $\operatorname{GF}\left(q^{m}\right)$ as $A_{\mathrm{R}}\left(q^{m}, n, d, r\right)$. If $C$ is an $(n, d, r)$ CRC over $\operatorname{GF}\left(q^{m}\right)$, then the code obtained by transposing all the expansion matrices of codewords in $C$ forms an $(m, d, r) \operatorname{CRC}$ over $\mathrm{GF}\left(q^{n}\right)$ with the same cardinality, and vice versa2 Therefore $A_{\mathrm{R}}\left(q^{m}, n, d, r\right)=A_{\mathrm{R}}\left(q^{n}, m, d, r\right)$, and henceforth in this paper we assume $n \leq m$ without loss of generality. We further observe that $A_{\mathrm{R}}\left(q^{m}, n, d, r\right)$ is a non-decreasing function of $m$ and $n$, and a nonincreasing function of $d$, and that $A_{\mathrm{S}}(q, n, 2 d, r)$ is a non-decreasing function of $n$ and a non-increasing function of $d$.

Proposition 5: For all $q, 2 \leq d \leq r \leq n \leq m$, and any $0 \leq p \leq r$,

$$
\min \left\{A_{\mathrm{S}}(q, n, 2(d+2 p), r), A_{\mathrm{S}}(q, m, 2(r-p), r)\right\} \leq A_{\mathrm{R}}\left(q^{m}, n, d+r, r\right) \leq A_{\mathrm{S}}(q, n, 2 d, r) .
$$

Also,

$$
A_{\mathrm{R}}\left(q^{m}, n, d+r, r\right) \geq A_{\mathrm{S}}(q, n, d+r, r) .
$$

Proof: Using the monotone properties of $A_{\mathrm{R}}\left(q^{m}, n, d_{\mathrm{R}}, r\right)$ and $A_{\mathrm{S}}\left(q, n, d_{\mathrm{S}}, r\right)$ above, the upper bound in (8) follows Proposition 2, while the lower bound in (8) follows Proposition 3 for $d_{\mathcal{C}}=2(r-p)$ and $d_{\mathcal{R}}=2(d+2 p)$. Finally, (9) follows Proposition 4 and the monotone properties of $A_{\mathrm{R}}\left(q^{m}, n, d_{\mathrm{R}}, r\right)$.

We remark that the lower bound in (8) is trivial for $d+2 p>\min \{r, n-r\}$ or $r-p>\min \{r, m-r\}$. Therefore, the lower bound in (8) is nontrivial when $\max \{0,2 r-m\} \leq p \leq \frac{1}{2} \min \{r-d, n-r-d\}$.

\footnotetext{
${ }^{2}$ However, the linearity of codes is not preserved through transposition.
} 
Combining the bounds in (8), we obtain that the cardinalities of optimal CRCs over sufficiently large fields are equal to the cardinalities of CDCs with related distances. Furthermore, we show that optimal CDCs can be constructed from such optimal CRCs.

Theorem 2: For all $q, 2 r \leq n \leq m$, and $1 \leq d \leq r, A_{\mathrm{R}}\left(q^{m}, n, d+r, r\right)=A_{\mathrm{S}}(q, n, 2 d, r)$ if either $d=r$ or $m \geq m_{0}$, where $m_{0}=(n-r)(r-d+1)+r+1$. Furthermore, if $C$ is an $(n, d+r, r)$ optimal CRC over $\operatorname{GF}\left(q^{m}\right)$ for $m \geq m_{0}$ or $d=r$, then $\mathcal{R}(C)$ is an optimal $(n, 2 d, r) \operatorname{CDC}$ over $\operatorname{GF}(q)$.

Proof: First, the case where $d=r$ directly follows (8) for $p=0$. Second, if $d<r$ and $m \geq m_{0}$, by (5) we obtain $A_{\mathrm{S}}(q, m, 2 r, r) \geq q^{m-r} \geq q^{m_{0}-r}$. Also, by [35, Lemma 1], we obtain $q^{r(r-d+1)-1}<$ $\alpha(r, r-d+1) \leq q^{r(r-d+1)}$ for all $2 \leq d<r$, and hence (5) yields $A_{\mathrm{S}}(q, n, 2 d, r)<q^{(n-r)(r-d+1)+1}=$ $q^{m_{0}-r} \leq A_{\mathrm{S}}(q, m, 2 r, r)$. Thus, when $p=0$, the lower bound in $(8)$ simplifies to $A_{\mathrm{R}}\left(q^{m}, n, d+r, r\right) \geq$ $A_{\mathrm{S}}(q, n, 2 d, r)$. Combining with the upper bound in (8), we obtain $A_{\mathrm{R}}\left(q^{m}, n, d+r, r\right)=A_{\mathrm{s}}(q, n, 2 d, r)$.

The second claim immediately follows Proposition 2 Theorem 2 implies that to determine $A_{\mathrm{S}}(q, n, 2 d, r)$ and to construct optimal CDCs, it is sufficient to determine $A_{\mathrm{R}}\left(q^{m}, n, d+r, r\right)$ and to construct optimal CRCs over an extension field sufficiently large. We observe that this implies that $A_{\mathrm{R}}\left(q^{m}, n, d+r, r\right)$ remains constant for all $m \geq m_{0}$. When $d=r$, $A_{\mathrm{R}}\left(q^{m}, n, 2 r, r\right)$ remains constant for $m \geq n$. When $d=1, m_{0}=(n-r+1) r+1$, but $A_{\mathrm{R}}\left(q^{m}, n, r+1, r\right)$ remains constant for $m \geq n$, and this is shown in Section IV-C

\section{CONSTANT-RANK CODES}

Having proved that optimal CRCs over sufficiently large extension fields lead to optimal CDCs, in this section we investigate the properties of CRCs.

\section{A. Graph-theoretic results for constant-rank codes}

We now define two families of graphs which are instrumental in our analysis of CRCs.

Definition 5: The bilinear forms graph $R_{q}(m, n, d)$ has as vertices all the vectors in $\operatorname{GF}\left(q^{m}\right)^{n}$ and two vertices $\mathbf{x}$ and $\mathbf{y}$ are adjacent if and only if $d_{\mathrm{R}}(\mathbf{x}, \mathbf{y})<d$. The constant-rank graph $K_{q}(m, n, d, r)$ is the subgraph of $R_{q}(m, n, d)$ induced by the vectors in $\operatorname{GF}\left(q^{m}\right)^{n}$ with rank $r$.

The orders of the bilinear forms and constant-rank graphs are thus given by $\left|R_{q}(m, n, d)\right|=q^{m n}$ and $\left|K_{q}(m, n, d, r)\right|=N_{r}\left(q^{m}, n\right)$. An independent set of $R_{q}(m, n, d)$ corresponds to a code with minimum rank distance $\geq d$. Due to the existence of MRD codes for all parameter values [39], we have $\alpha\left(R_{q}(m, n, d)\right)=q^{m(n-d+1)}$. Similarly, an independent set of $K_{q}(m, n, d, r)$ corresponds to a CRC with minimum rank distance $\geq d$, and hence $\alpha\left(K_{q}(m, n, d, r)\right)=A_{\mathrm{R}}\left(q^{m}, n, d, r\right)$. 
Lemma 2: The bilinear forms graph $R_{q}(m, n, d)$ is vertex transitive for all $q, m, n$, and $d$. The constantrank graph $K_{q}(m, m, d, m)$ is vertex transitive for all $q, m$, and $d$.

Proof: For given $\mathbf{u}, \mathbf{v} \in \mathrm{GF}\left(q^{m}\right)^{n}$, define $\phi(\mathbf{x})=\mathbf{x}+\mathbf{v}-\mathbf{u}$ for all $\mathbf{x} \in \operatorname{GF}\left(q^{m}\right)^{n}$. It is easily shown that $\phi$ is a graph automorphism of $R_{q}(m, n, d)$ satisfying $\phi(\mathbf{u})=\mathbf{v}$. By Definition $3, R_{q}(m, n, d)$ is hence vertex transitive.

Let $\mathbf{u}, \mathbf{v} \in \operatorname{GF}\left(q^{m}\right)^{m}$ have rank $m$, and denote their expansions as $\mathbf{U}$ and $\mathbf{V}$, respectively. For all $\mathbf{x} \in \operatorname{GF}\left(q^{m}\right)^{m}$ with rank $m$, define $\phi(\mathbf{x})=\mathbf{y}$ such that $\mathbf{Y}=\mathbf{X U}^{-1} \mathbf{V}$, where $\mathbf{X}$ and $\mathbf{Y}$ are the expansions of $\mathbf{x}$ and $\mathbf{y}$, respectively. We have $\phi(\mathbf{u})=\mathbf{v}, \operatorname{rk}(\phi(\mathbf{x}))=m$, and for all $\mathbf{x}, \mathbf{z} \in \mathrm{GF}\left(q^{m}\right)^{m}$, $d_{\mathrm{R}}(\phi(\mathbf{x}), \phi(\mathbf{z}))=\operatorname{rk}\left(\mathbf{X} \mathbf{U}^{-1} \mathbf{V}-\mathbf{Z} \mathbf{U}^{-1} \mathbf{V}\right)=\operatorname{rk}(\mathbf{X}-\mathbf{Z})=d_{\mathrm{R}}(\mathbf{x}, \mathbf{z})$. By Definition $2, \phi$ is an automorphism which maps $\mathbf{u}$ to $\mathbf{v}$ and hence $K_{q}(m, m, d, m)$ is vertex transitive.

It is worth noting that $K_{q}(m, n, d, r)$ is not vertex transitive in general.

\section{B. Bounds}

We now derive bounds on the maximum cardinality of CRCs. We first remark that the bounds on $A_{\mathrm{R}}\left(q^{m}, n, d, r\right)$ derived in Section $\amalg$ can be used in this section. Also, since $A_{\mathrm{R}}\left(q^{m}, n, 1, r\right)=N_{r}\left(q^{m}, n\right)$ and $A_{\mathrm{R}}\left(q^{m}, n, d, r\right)=1$ for $d>2 r$, we shall assume $2 \leq d \leq 2 r$ henceforth.

We first derive the counterparts of the Gilbert and the Hamming bounds for CRCs in terms of intersections of spheres with rank radii.

Proposition 6: For all $q, 1 \leq r, d \leq n \leq m$, and $t \stackrel{\text { def }}{=}\left\lfloor\frac{d-1}{2}\right\rfloor$,

$$
\frac{N_{r}\left(q^{m}, n\right)}{\sum_{i=0}^{d-1} J\left(q^{m}, n, i, r, r\right)} \leq A_{\mathrm{R}}\left(q^{m}, n, d, r\right) \leq \frac{N_{r}\left(q^{m}, n\right)}{\sum_{i=0}^{t} J\left(q^{m}, n, i, r, r\right)} .
$$

The proof is straightforward and hence omitted. The Hamming bound is generalized as follows.

Proposition 7: For all $q, 1 \leq r, d, s \leq n \leq m$, and $t \stackrel{\text { def }}{=}\left\lfloor\frac{d-1}{2}\right\rfloor$,

$$
A_{\mathrm{R}}\left(q^{m}, n, d, r\right) \leq \frac{N_{s}\left(q^{m}, n\right)}{\sum_{i=0}^{t} J\left(q^{m}, n, i, s, r\right)} .
$$

Proof: Let $C=\left\{\mathbf{c}_{k}\right\}_{k=0}^{K-1}$ be an $(n, d, r)$ CRC over $\operatorname{GF}\left(q^{m}\right)$. For all $0 \leq k \leq K-1$ and $0 \leq s \leq n-1$, if we denote the set of vectors in $\mathrm{GF}\left(q^{m}\right)^{n}$ with rank $s$ and distance $\leq t$ from $\mathbf{c}_{k}$ as $R_{k, s}$, then $\left|R_{k, s}\right|=\sum_{i=0}^{t} J(i, s, r)$ for all $k$. Clearly $R_{k, s} \cap R_{l, s}=\emptyset$ for all $k \neq l$, and hence $N_{s} \geq\left|\bigcup_{k=0}^{K-1} R_{k, s}\right|=K\left|R_{k, s}\right|$, which yields (11).

We now derive upper bounds on $A_{\mathrm{R}}\left(q^{m}, n, d, r\right)$. We begin by proving the counterpart in rank metric codes of a well-known bound on constant-weight codes proved by Johnson in [40]. 
Proposition 8: For all $q, 1 \leq r, d<n \leq m$,

$$
\begin{aligned}
A_{\mathrm{R}}\left(q^{m}, m, d, m\right) & \leq q^{m-1}\left(q^{m}-1\right) A_{\mathrm{R}}\left(q^{m-1}, m-1, d, m-1\right) \\
A_{\mathrm{R}}\left(q^{m}, n, d, r\right) & \leq \frac{q^{n}-1}{q^{n-r}-1} A_{\mathrm{R}}\left(q^{m}, n-1, d, r\right) .
\end{aligned}
$$

The proof of Proposition 8 is given in Appendix B.

The Singleton bound for rank metric codes yields upper bounds on $A_{\mathrm{R}}\left(q^{m}, n, d, r\right)$. For any $I \subseteq$ $\{0,1, \ldots, n\}$, let $A_{\mathrm{R}}\left(q^{m}, n, d, I\right)$ denote the maximum cardinality of a code of length $n$ and minimum rank distance $d$ over $\operatorname{GF}\left(q^{m}\right)$ such that all codewords have rank weights belonging to $I$.

Proposition 9: For all $q, 1 \leq r, d \leq n \leq m$, define $P_{r} \stackrel{\text { def }}{=}\{i: 0 \leq i \leq n,|i-r| \geq d\}$ and $Q_{r, a} \stackrel{\text { def }}{=} P_{r} \cap\{a+k d: k \in \mathbb{Z}\}$ for all $0 \leq a<d$. Then

$$
\begin{aligned}
A_{\mathrm{R}}\left(q^{m}, n, d, r\right) & \leq q^{m(n-d+1)}-A_{\mathrm{R}}\left(q^{m}, n, d, P_{r}\right) \\
& \leq q^{m(n-d+1)}-\max \left\{\sum_{i \in P_{r}} M\left(q^{m}, n, d, i\right), \sum_{j \in Q_{r, a}} A_{\mathrm{R}}\left(q^{m}, n, d, j\right)\right\} .
\end{aligned}
$$

Proof: For $0 \leq j \leq n$, let $C_{j}$ be an optimal $(n, d, j)$ CRC and let $C \subseteq \operatorname{GF}\left(q^{m}\right)^{n}$ be a code with minimum rank distance $d$ and whose codewords have rank weights belonging to $P_{r}$. Eq. (14) directly follows the Singleton bound on $C \dot{\cup} C_{r}$, where $\dot{\cup}$ denotes disjoint union. Let $G$ be an $(n, n-d+1, d)$ linear MRD code over $\operatorname{GF}\left(q^{m}\right)$, and denote the subset of codewords with ranks belonging to $P_{r}$ as $G^{\prime}$. Finally define $C^{\prime} \stackrel{\text { def }}{=} \bigcup_{j \in Q_{r, a}} C_{j}$. Both $G^{\prime}$ and $C^{\prime}$ are codes in $\operatorname{GF}\left(q^{m}\right)^{n}$ with minimum rank distance $d$ and whose codewords have rank weights belonging to $P_{r}$, hence $A_{\mathrm{R}}\left(q^{m}, n, P_{r}\right) \geq \max \left\{\left|G^{\prime}\right|,\left|C^{\prime}\right|\right\}$, which leads to (15).

We now determine the counterpart of the Singleton bound for CRCs.

Proposition 10: For all $0 \leq i \leq \min \{d-1, r\}, J_{i} \stackrel{\text { def }}{=}\{r-i, r-i+1, \ldots, \min \{n-i, r\}\}$. Then

$$
\begin{aligned}
A_{\mathrm{R}}\left(q^{m}, n, d, r\right) & \leq A_{\mathrm{R}}\left(q^{m}, n-i, d-i, J_{i}\right) \\
& \leq \sum_{j=r-i}^{\min \{n-i, r\}} A_{\mathrm{R}}\left(q^{m}, n-i, d-i, j\right) .
\end{aligned}
$$

Proof: Let $C$ be an optimal $(n, d, r)$ CRC over $\operatorname{GF}\left(q^{m}\right)$, and consider the code $C_{i}$ obtained by puncturing $i$ coordinates of the codewords in $C$. Since $i \leq r$, the codewords of $C_{i}$ all have ranks between $r-i$ and $\min \{n-i, r\}$. Also, since $i<d$, any two codewords have distinct puncturings, and we obtain $\left|C_{i}\right|=|C|$ and $d_{\mathrm{R}}\left(C_{i}\right) \geq d-i$. Hence $A_{\mathrm{R}}\left(q^{m}, n, d, r\right)=|C|=\left|C_{i}\right| \leq A_{\mathrm{R}}\left(q^{m}, n-i, d-i, J_{i}\right)$. (17) directly follows a union bound on $A_{\mathrm{R}}\left(q^{m}, n-i, d-i, J_{i}\right)$.

We now combine the counterparts of the Johnson bound in (13) and of the Singleton bound in Proposition 10 in order to obtain an upper bound on $A_{\mathrm{R}}\left(q^{m}, n, d, r\right)$ for $d \leq r$. 
Proposition 11: For all $q, 1 \leq d \leq r \leq n \leq m, A_{\mathrm{R}}\left(q^{m}, n, d, r\right) \leq\left[\begin{array}{l}n \\ r\end{array}\right] \alpha(m, r-d+1)$.

Proof: Applying (13) $n-r$ times successively, we obtain $A_{\mathrm{R}}\left(q^{m}, n, d, r\right) \leq\left[\begin{array}{c}n \\ r\end{array}\right] A_{\mathrm{R}}\left(q^{m}, r, d, r\right)$. For $n=r$ and $i=d-1, J_{i}=\{r-d+1\}$ and hence (16) yields $A_{\mathrm{R}}\left(q^{m}, r, d, r\right) \leq A_{\mathrm{R}}\left(q^{m}, r-d+1,1, r-\right.$ $d+1)=N_{r-d+1}\left(q^{m}, r-d+1\right)=\alpha(m, r-d+1)$. Thus $A_{\mathrm{R}}\left(q^{m}, n, d, r\right) \leq\left[\begin{array}{l}n \\ r\end{array}\right] \alpha(m, r-d+1)$.

We now derive the counterpart in rank metric codes of the Bassalygo-Elias bound [41]. We also tighten the bound when $d>r+1$.

Proposition 12: For $\max \{r, d\} \leq k \leq n, 0 \leq s \leq k, k \leq l \leq m$, and any code $C \subseteq \mathrm{GF}\left(q^{l}\right)^{k}$ with minimum rank distance $d$ and rank weight distribution $A_{i} \stackrel{\text { def }}{=}|\{\mathbf{c} \in C: \operatorname{rk}(\mathbf{c})=i\}|$,

$$
A_{\mathrm{R}}\left(q^{m}, n, d, r\right) \geq \max _{s,\left\{A_{i}\right\}, k, l} \frac{\sum_{i=0}^{n} A_{i} J\left(q^{l}, k, s, r, i\right)}{N_{s}\left(q^{l}, k\right)} .
$$

Furthermore, if $r+1<d \leq 2 r$, then

$$
A_{\mathrm{R}}\left(q^{m}, n, d, r\right) \geq \max _{s,\left\{A_{i}\right\}, k, l} \frac{\sum_{i=0}^{n} A_{i} J\left(q^{l}, k, s, r, i\right)}{N_{s}\left(q^{l}, k\right)-\sum_{i=0}^{n} A_{i} \sum_{t=0}^{d-r-1} J\left(q^{l}, k, s, t, i\right)} .
$$

The proof of Proposition 12 is given in Appendix $\mathrm{C}$

Although the RHS of (18) and (19) can be maximized over $\left\{A_{i}\right\}$, it is difficult to do so since $\left\{A_{i}\right\}$ is not available for most rank metric codes with the exception of linear MRD codes. Thus, we derive a bound using the rank weight distribution of linear MRD codes.

Corollary 1: For all $q, 1 \leq r, d \leq n \leq m$,

$$
A_{\mathrm{R}}\left(q^{m}, n, d, r\right) \geq N_{r}\left(q^{m}, n\right) q^{m(-d+1)} .
$$

Proof: Applying (18) to an $(n, n-d+1, d)$ MRD code over $\operatorname{GF}\left(q^{m}\right)$, we obtain $N_{s}\left(q^{m}, n\right) A_{\mathrm{R}}\left(q^{m}, n, d, r\right) \geq$ $\sum_{i=0}^{n} M\left(q^{m}, n, d, i\right) J\left(q^{m}, n, s, r, i\right)$. Summing for all $0 \leq s \leq n$, we obtain (20) since $\sum_{s=0}^{n} J(s, r, i)=$ $N_{r}\left(q^{m}, n\right)$.

We also give an alternate proof of (20) based on the results in Section IV-A Since $K_{q}(m, n, d, r)$ is a subgraph of $R_{q}(m, n, d)$, the inclusion map is a trivial homomorphism from $K_{q}(m, n, d, r)$ to $R_{q}(m, n, d)$. By Lemma 2, $R_{q}(m, n, d)$ is vertex transitive. We hence apply (6) to these graphs, which yields (20).

The RHS of (18) and (19) decrease rapidly with increasing $d$, rendering the bounds trivial for $d$ approaching $2 r$.

We investigate below the tightness of the bound in Corollary 1

Proposition 13: For all $q, 2 \leq d \leq r \leq n \leq m$, let $C\left(q^{m}, n, d, r\right) \stackrel{\text { def }}{=} A_{\mathrm{R}}\left(q^{m}, n, d, r\right) /\left[N_{r}\left(q^{m}, n\right) q^{m(-d+1)}\right]$. Then

$$
\begin{aligned}
& C\left(q^{m}, n, d, r\right) \leq \frac{q^{2}}{q^{2}-1} \text { for } r+d-1 \leq m \\
& C\left(q^{m}, n, d, r\right)<\frac{q-1}{q} K_{q}^{-1} \quad \text { otherwise },
\end{aligned}
$$


where $K_{q} \stackrel{\text { def }}{=} \prod_{j=1}^{\infty}\left(1-q^{-j}\right)$.

Proof: By Proposition 11, $C\left(q^{m}, n, d, r\right) \leq q^{m(d-1)} \alpha(m, r-d+1) / \alpha(m, r)=q^{(m-r+d-1)(d-1)} / \alpha(m-$ $r+d-1, d-1)$. Since $\alpha(n, l)>\frac{q}{q-1} K_{q} q^{n l}$ for all $1 \leq l \leq n-1$ [35, Lemma 1], we obtain $C\left(q^{m}, n, d, r\right) \leq \frac{q-1}{q} K_{q}^{-1}$. Finally, $\alpha(n, l) \geq \frac{q^{2}-1}{q^{2}} q^{n l}$ for $2 l \leq n$ [35, Lemma 1] yields (21).

It is worth noting that $K_{q}$ above represents the fraction of invertible $m \times m$ matrices over $\operatorname{GF}(q)$ as $m$ approaches infinity. $K_{q}^{-1}$ decreases with $q$ and satisfies $1<K_{q}^{-1} \leq K_{2}^{-1}<4$. Thus the bound in Corollary 1 is tight up to a scalar when $d \leq r$. We also remark that the bound in (21) is tighter than that in (22). However, these bounds are not constructive. Below we derive constructive bounds on $A_{\mathrm{R}}\left(q^{m}, n, d, r\right)$.

\section{Constructive bounds}

We now give explicit constructions of good CRCs when $d \leq r$, which in turn yield asymptotically tight lower bounds on $A_{\mathrm{R}}\left(q^{m}, n, d, r\right)$.

Proposition 14: For all $q, 2 \leq d \leq r \leq n \leq m, A_{\mathrm{R}}\left(q^{m}, n, d, r\right) \geq M\left(q^{m}, n, d, r\right)>\left[\begin{array}{l}n \\ r\end{array}\right] q^{m(r-d)}$.

Proof: The codewords of rank $r$ in an $(n, n-d+1, d)$ linear MRD code over $\operatorname{GF}\left(q^{m}\right)$ form an $(n, d, r)$ CRC. Thus, $A_{\mathrm{R}}\left(q^{m}, n, d, r\right) \geq M\left(q^{m}, n, d, r\right)$.

We now prove the lower bound on $M\left(q^{m}, n, d, r\right)$. First, for $d=r, M\left(q^{m}, n, r, r\right)=\left[\begin{array}{l}n \\ r\end{array}\right]\left(q^{m}-\right.$ 1) $>\left[\begin{array}{l}n \\ r\end{array}\right]$. Second, suppose $d<r$. By (3), $M\left(q^{m}, n, d, r\right)$ can be expressed as $M\left(q^{m}, n, d, r\right)=$ $\left[\begin{array}{l}n \\ r\end{array}\right] \sum_{j=d}^{r}(-1)^{r-j} \mu_{j}$, where $\mu_{j} \stackrel{\text { def }}{=} q^{(r-j)(r-j-1) / 2}\left[\begin{array}{c}r \\ j\end{array}\right]\left(q^{m(j-d+1)}-1\right)$. It can be easily shown that $\mu_{j}>$ $\mu_{j-1}$ for $d+1 \leq j \leq r$, and hence $M\left(q^{m}, n, d, r\right) \geq\left[\begin{array}{l}n \\ r\end{array}\right]\left(\mu_{r}-\mu_{r-1}\right)$. Therefore, $M\left(q^{m}, n, d, r\right) \geq$ $\left[\begin{array}{l}n \\ r\end{array}\right]\left[\left(q^{m(r-d+1)}-1\right)-\left[\begin{array}{l}r \\ 1\end{array}\right]\left(q^{m(r-d)}-1\right)\right]>\left[\begin{array}{l}n \\ r\end{array}\right] q^{m(r-d)}$.

Corollary 2: For all $q, 1 \leq r \leq n \leq m, A_{\mathrm{R}}\left(q^{m}, n, r, r\right)=\left[\begin{array}{c}n \\ r\end{array}\right]\left(q^{m}-1\right)$.

Proof: By Proposition 11, $A_{\mathrm{R}}\left(q^{m}, n, r, r\right) \leq\left[\begin{array}{c}n \\ r\end{array}\right]\left(q^{m}-1\right)$, and by Proposition 14, $A_{\mathrm{R}}\left(q^{m}, n, r, r\right) \geq$ $M\left(q^{m}, n, r, r\right)=\left[\begin{array}{c}n \\ r\end{array}\right]\left(q^{m}-1\right)$.

By Corollary 2, the codewords of rank $r$ in an $(n, n-r+1, r)$ linear MRD code are optimal. We investigate below the tightness of the constructive lower bound in Proposition 14

Proposition 15: For all $q, 1 \leq d<r \leq n \leq m$, let $B\left(q^{m}, n, d, r\right) \stackrel{\text { def }}{=} A_{\mathrm{R}}\left(q^{m}, n, d, r\right) / M\left(q^{m}, n, d, r\right)$. 
Then for $m \geq 3$,

$$
\begin{aligned}
B\left(2^{m}, m, m-1, m\right) & \leq 2^{m-1}-1 \\
B\left(q^{m}, m, m-1, m\right) & <\frac{q-1}{q-2} \quad \text { for } q>2 \\
B\left(q^{m}, m, m-2, m\right) & <\frac{\left(q^{2}-1\right)(q-1)}{\left(q^{2}-1\right)(q-2)+1} \\
B\left(q^{m}, m, d, m\right) & <\frac{\left(q^{3}-1\right)\left(q^{2}-1\right)(q-1)}{\left(q^{3}-1\right)\left(q^{2}-1\right)(q-2)+q^{3}-2} \quad \text { for } d<m-2 \\
B\left(q^{m}, n, d, r\right) & <\frac{q}{q-1} \text { for } r<m .
\end{aligned}
$$

The proof of Proposition 15 is given in Appendix D

Proposition 15 shows that for all but one cases, the codewords of rank $r$ in an $(n, n-d+1, d)$ MRD code form a code whose cardinality is very close to that of an optimal CRC, especially when $q$ is large.

We now construct $(n, d, r)$ CRCs for $d>r$ using generalized Gabidulin codes [30]. Let $\mathbf{g} \in \operatorname{GF}\left(q^{m}\right)^{n}$ have rank $n$, and for $0 \leq i \leq m-1$, denote the vector in $\operatorname{GF}\left(q^{m}\right)^{n}$ obtained by elevating each coordinate of $\mathbf{g}$ to the $q^{a i}$-th power as $\mathbf{g}^{[i]}$, where $a$ and $m$ are coprime. Let $\mathcal{C}$ be the $(n, n-d+1, d)$ generalized Gabidulin code over $\operatorname{GF}\left(q^{m}\right)$ generated by $\left(\mathbf{g}^{[0]^{T}} \mathbf{g}^{[1]^{T}} \cdots \mathbf{g}^{[n-d]^{T}}\right)^{T}$, and $\mathcal{C}^{\prime}$ be the $(n, d-r, n-d+$ $r+1)$ generalized Gabidulin code generated by $\left(\mathbf{g}^{[n-d+1]^{T}} \mathbf{g}^{[n-d+2]^{T}} \cdots \mathbf{g}^{[n-r]^{T}}\right)^{T}$. We consider the coset $\mathcal{C}+\mathbf{c}^{\prime}$, where $\mathbf{c}^{\prime} \in \mathcal{C}^{\prime}$, and we denote the number of codewords of rank $r$ in $\mathcal{C}+\mathbf{c}^{\prime}$ as $\sigma_{r}\left(\mathbf{c}^{\prime}\right)$.

Lemma 3: For all $d>r$, there exists $\mathbf{c}^{\prime} \in \mathcal{C}^{\prime}$ such that $\sigma_{r}\left(\mathbf{c}^{\prime}\right) \geq\left[\begin{array}{l}n \\ r\end{array}\right] q^{m(r-d+1)}$.

Proof: Any codeword $\mathbf{c}^{\prime} \in C^{\prime}$ can be expressed as $\mathbf{c}^{\prime}=c_{n-d+1} \mathbf{g}^{[n-d+1]}+c_{n-d+2} \mathbf{g}^{[n-d+2]}+\ldots+$ $c_{n-r} \mathbf{g}^{[n-r]}$, where $c_{i} \in \operatorname{GF}\left(q^{m}\right)$ for $n-d+1 \leq i \leq n-r$. If $c_{n-r}=0$, then $\left(\mathcal{C}+\mathbf{c}^{\prime}\right) \subset \mathcal{D}$, where $\mathcal{D}$ is the $(n, n-r, r+1)$ generalized Gabidulin code generated by $\left(\mathbf{g}^{[0]^{T}} \mathbf{g}^{[1]^{T}} \cdots \mathbf{g}^{[n-r-1]^{T}}\right)^{T}$. Therefore $\sigma_{r}\left(\mathbf{c}^{\prime}\right)=0$ if $c_{n-r}=0$.

Denote the number of codewords of rank $r$ in $\mathcal{C} \oplus \mathcal{C}^{\prime}$ as $\tau_{r}$. Since $\bigcup_{\mathbf{c}^{\prime} \in \mathcal{C}^{\prime}}\left(\mathcal{C}+\mathbf{c}^{\prime}\right)=\mathcal{C} \oplus \mathcal{C}^{\prime}$, we have $\tau_{r}=\sum_{\mathbf{c}^{\prime} \in \mathcal{C}^{\prime}} \sigma_{r}\left(\mathbf{c}^{\prime}\right)$. Also, $\mathcal{C} \oplus \mathcal{C}^{\prime}$ forms an $(n, n-r+1, r)$ MRD code, and hence $\tau_{r}=M\left(q^{m}, n, r, r\right)=$ $\left[\begin{array}{l}n \\ r\end{array}\right]\left(q^{m}-1\right)$. Suppose that for all $\mathbf{c}^{\prime} \in \mathcal{C}^{\prime}, \sigma_{r}\left(\mathbf{c}^{\prime}\right)<\left[\begin{array}{l}n \\ r\end{array}\right] q^{m(r-d+1)}$. Then $\tau_{r}=\sum_{\mathbf{c}^{\prime}: c_{n-r} \neq 0} \sigma_{r}\left(\mathbf{c}^{\prime}\right)<$ $\left[\begin{array}{l}n \\ r\end{array}\right]\left(q^{m}-1\right)$, which contradicts $\tau_{r}=\left[\begin{array}{l}n \\ r\end{array}\right]\left(q^{m}-1\right)$.

Although Lemma 3 proves the existence of a vector $\mathbf{c}^{\prime}$ for which the translate $\mathcal{C}+\mathbf{c}^{\prime}$ has high cardinality, it does not indicate how to choose $\mathbf{c}^{\prime}$. For $d=r+1$, it can be shown that all $\mathbf{c}^{\prime} \in \mathcal{C}^{\prime}$ satisfy the bound, and that they all lead to optimal codes.

Corollary 3: If $d=r+1$, then $\sigma_{r}\left(\mathbf{c}^{\prime}\right)=\left[\begin{array}{l}n \\ r\end{array}\right]$ for all $\mathbf{c}^{\prime} \in \mathcal{C}^{\prime}$.

Proof: First, by Proposition 5, $\sigma_{r}\left(\mathbf{c}^{\prime}\right) \leq A_{\mathrm{R}}\left(q^{m}, n, r+1, r\right) \leq A_{\mathrm{S}}(q, n, 2, r)=\left[\begin{array}{l}n \\ r\end{array}\right]$ for all $\mathbf{c}^{\prime} \in \mathcal{C}^{\prime}$. Suppose there exists $\mathbf{c}^{\prime}$ such that $\sigma_{r}\left(\mathbf{c}^{\prime}\right)<\left[\begin{array}{l}n \\ r\end{array}\right]$. Then $\tau_{r}<\left[\begin{array}{l}n \\ r\end{array}\right]\left(q^{m}-1\right)$, which contradicts $\tau_{r}=\left[\begin{array}{l}n \\ r\end{array}\right]\left(q^{m}-1\right)$. 
Proposition 16: For all $q, 1 \leq r<d \leq n \leq m, A_{\mathrm{R}}\left(q^{m}, n, d, r\right) \geq\left[\begin{array}{l}n \\ r\end{array}\right] q^{n(r-d+1)}$, and a class of codes that satisfy this bound can be constructed from Lemma 3 .

Proof: The codewords of rank $r$ in a code considered in Lemma 3 form an $(n, d, r)$ CRC over $\mathrm{GF}\left(q^{m}\right)$ with cardinality $\geq\left[\begin{array}{l}n \\ r\end{array}\right] q^{m(r-d+1)}$. Therefore, $A_{\mathrm{R}}\left(q^{m}, n, d, r\right) \geq\left[\begin{array}{l}n \\ r\end{array}\right] q^{m(r-d+1)}$. The proof is concluded by noting that $A_{\mathrm{R}}\left(q^{m}, n, d, r\right) \geq A_{\mathrm{R}}\left(q^{n}, n, d, r\right) \geq\left[\begin{array}{l}n \\ r\end{array}\right] q^{n(r-d+1)}$.

Corollary 4: For all $q, 1 \leq r<n \leq m, A_{\mathrm{R}}\left(q^{m}, n, r+1, r\right)=\left[\begin{array}{l}n \\ r\end{array}\right]=A_{\mathrm{S}}(q, n, 2, r)$.

Proof: Combine Proposition 5 and Proposition 16.

We note that $\left[\begin{array}{l}n \\ r\end{array}\right]$ is independent of $m$. We also remark that the lower bound in Proposition 16 is also trivial for $d$ approaching $2 r$. Since the proof is only partly constructive, computer search can be used to help find better results for small parameter values.

\section{Asymptotic results}

In this section, we study the asymptotic behavior of CRCs using the following set of normalized parameters: $\nu=\frac{n}{m}, \rho=\frac{r}{m}$, and $\delta=\frac{d}{m}$. By definition, $0 \leq \rho, \delta \leq \nu$, and since we assume $n \leq m$, $\nu \leq 1$. We consider the asymptotic rate defined as $a_{\mathrm{R}}(\nu, \delta, \rho) \stackrel{\text { def }}{=} \lim _{m \rightarrow \infty} \sup \left[\log _{q^{m^{2}}} A_{\mathrm{R}}\left(q^{m}, n, d, r\right)\right]$. We now investigate how $A_{\mathrm{R}}\left(q^{m}, n, d, r\right)$ behaves as the parameters tend to infinity. Without loss of generality, we only consider the case where $0 \leq \delta \leq \min \{\nu, 2 \rho\}$, since $a_{\mathrm{R}}(\nu, \delta, \rho)=0$ for $\delta>2 \rho$.

Proposition 17: For $0 \leq \delta \leq \rho$,

$$
a_{\mathrm{R}}(\nu, \delta, \rho)=\rho(1+\nu-\rho)-\delta \text {. }
$$

For $\rho \leq \delta$, we have to distinguish three cases. First, for $2 \rho \leq \nu$,

$$
\max \left\{\frac{(1-\rho)(\nu-\rho)}{1+2 \nu-3 \rho}(2 \rho-\delta), \frac{\nu-\rho}{2}(2 \rho-\delta), \rho(2 \nu-\rho)-\nu \delta\right\} \leq a_{\mathrm{R}}(\nu, \delta, \rho) \leq(\nu-\rho)(2 \rho-\delta) .
$$

Second, for $\nu \leq 2 \rho \leq 1$,

$$
\max \left\{\frac{\rho(1-\rho)}{1+\rho}(\nu-\delta), \frac{\rho}{2}(2 \nu-2 \rho-\delta), \rho(2 \nu-\rho)-\nu \delta\right\} \leq a_{\mathrm{R}}(\nu, \delta, \rho) \leq \rho(\nu-\delta) .
$$

Third, for $2 \rho \geq 1$,

$$
\max \left\{\frac{\rho}{3}(2-4 \rho+\nu-\delta), \frac{\rho}{2}(2 \nu-2 \rho-\delta), \rho(2 \nu-\rho)-\nu \delta, 0\right\} \leq a_{\mathrm{R}}(\nu, \delta, \rho) \leq \rho(\nu-\delta) .
$$

The proof of Proposition 17 is given in Appendix E.

Proposition 17 indicates that the codewords of rank $r$ in an $(n, n-d+1, d)$ linear MRD code $(d \leq r)$ form an asymptotically optimal $(n, d, r)$ CRC. In particular, the set of codewords with rank $n$ constitutes 
a CRC of rank $n$ and asymptotic rate of $\nu-\delta$, which is equal to the asymptotic rate of an optimal rank metric code [42].

The bounds on $a_{\mathrm{R}}(\nu, \delta, \rho)$ given in Proposition 17 are illustrated in Figures 1, 2, and 3 for $\nu=3 / 4$ and $\rho=1 / 4, \rho=2 / 5$, and $\rho=3 / 5$, respectively. Note that these three parameters correspond to the three cases in (29), (30), and (31), respectively.

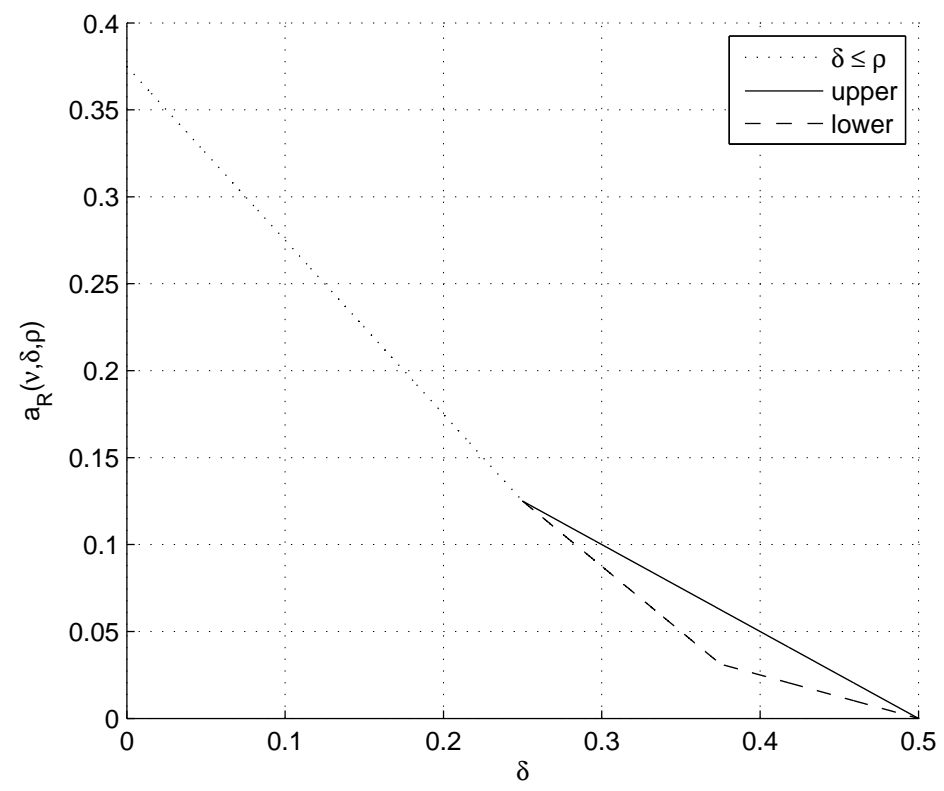

Fig. 1. Asymptotic bounds on the maximal rate of a CRC as a function of $\delta$, with $\nu=3 / 4$ and $\rho=1 / 4$.

In Figures 1, 2, and 3, we can split the range of $\delta$ into two regions: when $\delta \leq \rho$, the asymptotic rate of CRCs is determined due to the construction of good CRCs when $d \leq r$; when $\delta \geq \rho$, we only have bounds on the asymptotic rate of CRCs. We remark that in (29), the first two lower bounds are competing, thus forming a triangle-shaped region for $a_{\mathrm{R}}(\nu, \delta, \rho)$. However, for (30) and (31), the shape of the possible region for $a_{\mathrm{R}}(\nu, \delta, \rho)$ depends on both $\nu$ and $\rho$. Also, the lower bounds based on the connection between CDCs and CRCs (the first two lower bounds in the LHS of (29), (30), and (31)) are tighter for $2 \rho \leq \nu$ and on the other hand become trivial for $\rho$ approaching 1 . 


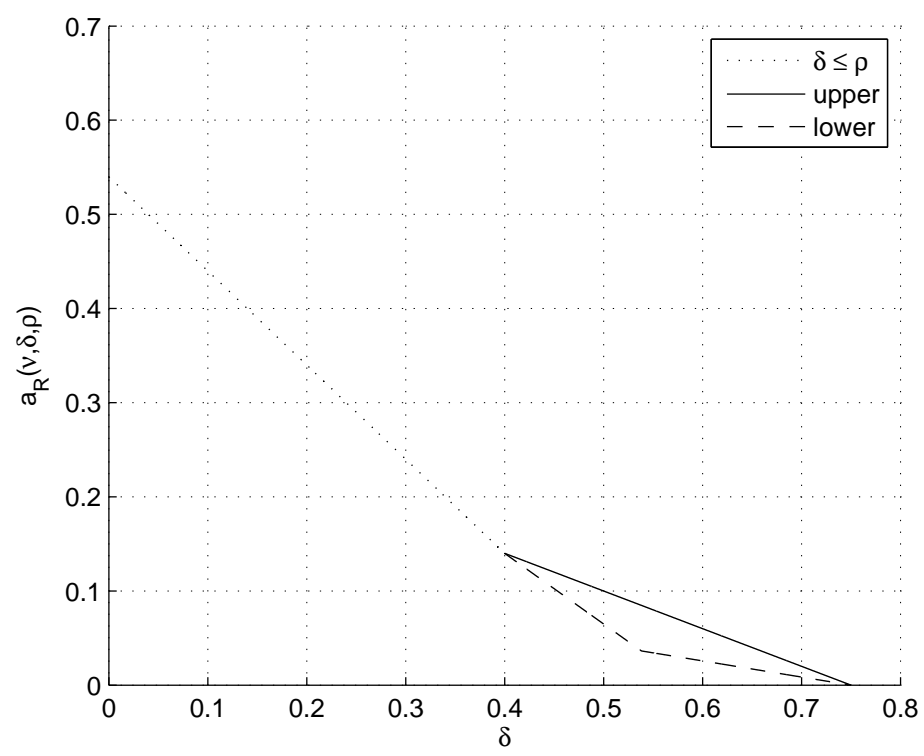

Fig. 2. Asymptotic bounds on the maximal rate of a CRC as a function of $\delta$, with $\nu=3 / 4$ and $\rho=2 / 5$.

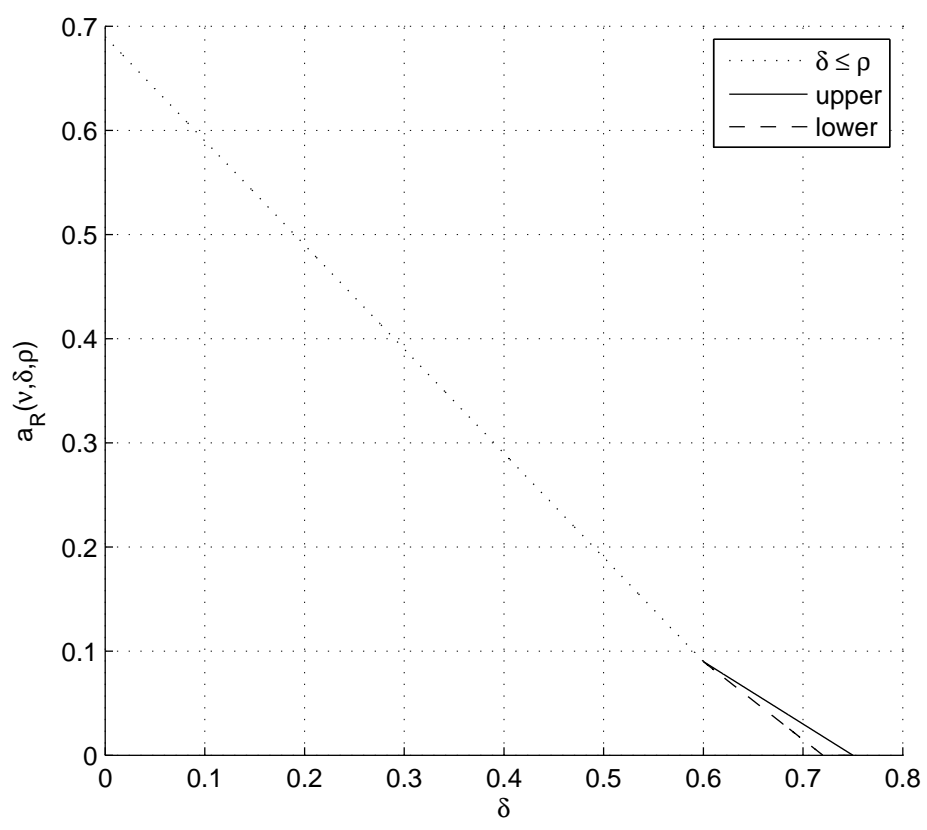

Fig. 3. Asymptotic bounds on the maximal rate of a CRC as a function of $\delta$, with $\nu=3 / 4$ and $\rho=3 / 5$. 
APPENDIX

\section{A. Proof of Proposition 4}

Proof: Let us first focus on $\operatorname{GF}\left(q^{n}\right)$ and consider a basis $B_{n}$ of $\operatorname{GF}\left(q^{n}\right)$ over $\operatorname{GF}(q)$. Define the code $C$ over $\operatorname{GF}\left(q^{n}\right)$ formed by the codewords $\mathbf{c}_{i} \stackrel{\text { def }}{=} \mathbf{b}_{i} \mathbf{H}_{i}$ for $0 \leq i \leq|\Delta|-1$, where the expansion of $\mathbf{b}_{i} \in \operatorname{GF}\left(q^{n}\right)^{r}$ is given by $\mathbf{H}_{i}^{T}$. Let us consider $\mathbf{c}_{i}=\mathbf{b}_{i} \mathbf{H}_{i}$ and $\mathbf{c}_{j}=\mathbf{b}_{j} \mathbf{H}_{j}$. Let $\beta_{i, k_{0}}, \beta_{i, k_{1}}, \ldots, \beta_{i, k_{d-1}}$ be coordinates of $\mathbf{b}_{i}$ linearly independent to the coordinates of $\mathbf{b}_{j}$ and $\beta_{j, l_{0}}, \beta_{j, l_{1}}, \ldots, \beta_{j, l_{d-1}}$ be coordinates of $\mathbf{b}_{j}$ linearly independent to the coordinates of $\mathbf{b}_{i}$. We thus define the basis

$$
\gamma_{i, j}=\left\{\beta_{i, k_{0}}, \beta_{i, k_{1}}, \ldots, \beta_{i, k_{d-1}}, \beta_{j, l_{0}}, \beta_{j, l_{1}}, \ldots, \beta_{j, l_{d-1}}, \gamma_{2 d}, \gamma_{2 d+1}, \ldots, \gamma_{n-1}\right\}
$$

of $\operatorname{GF}\left(q^{n}\right)$ over $\operatorname{GF}(q)$. Expanding $\mathbf{c}_{i}-\mathbf{c}_{j}$ with respect to the basis $\gamma_{i, j}$, we obtain $\operatorname{rk}\left(\mathbf{c}_{i}-\mathbf{c}_{j}\right) \geq$

$\operatorname{rk}\left(\overline{\mathbf{H}}_{i}^{T}-\overline{\mathbf{H}}_{j}^{T}\right)$, where $\overline{\mathbf{H}}_{i}$ denotes the $d$ rows of $\mathbf{H}_{i}$ corresponding to $\beta_{i, k_{0}}, \beta_{i, k_{1}}, \ldots, \beta_{i, k_{d-1}}$ and $\overline{\mathbf{H}}_{j}$ denotes the $d$ rows of $\mathbf{H}_{j}$ corresponding to $\beta_{j, l_{0}}, \beta_{j, l_{1}}, \ldots, \beta_{j, l_{d-1}}$. Thus

$$
\operatorname{rk}\left(\overline{\mathbf{H}}_{i}^{T}-\overline{\mathbf{H}}_{j}^{T}\right)=\operatorname{rk}\left(\beta_{i, k_{0}}, \beta_{i, k_{1}}, \ldots, \beta_{i, k_{d-1}},-\beta_{j, l_{0}},-\beta_{j, l_{1}}, \ldots,-\beta_{j, l_{d-1}}\right)=2 d .
$$

Using an argument similar to that in the proof of Prop. 3, we can show that $d_{\mathrm{R}} \leq d+r$.

For $m \geq n$ and a basis $B_{m}$ of $\operatorname{GF}\left(q^{m}\right)$ over $\operatorname{GF}(q)$, we append $m-n$ all-zero rows to the expansion with respect to $B_{n}$ of codewords in $C$, then the matrices are the expansions (with respect to $B_{m}$ ) of codewords of a CRC of length $n$ and rank $r$ over $\operatorname{GF}\left(q^{m}\right)$ and minimum rank distance $\geq 2 d$.

We now derive the bounds on the diameter $D_{\mathrm{R}}$ of $C$. Let $\mathbf{x}$ and $\mathbf{y}$ be distinct codewords in $C$ such that $d_{\mathrm{S}}(\mathcal{C}(\mathbf{x}), \mathcal{C}(\mathbf{y}))=d_{\mathrm{S}}(\mathcal{R}(\mathbf{x}), \mathcal{R}(\mathbf{y}))=D_{\mathrm{S}}$, then by Theorem 11 $\frac{1}{2} \max \left\{D_{\mathrm{S}}, 3 D_{\mathrm{S}}-2 r\right\} \leq D_{\mathrm{R}}$. Let $\mathbf{x}^{\prime}$ and $\mathbf{y}^{\prime}$ be distinct codewords in $C$ such that $d_{\mathrm{R}}\left(\mathbf{x}^{\prime}, \mathbf{y}^{\prime}\right)=D_{\mathrm{R}}$, then by Theorem $1, D_{\mathrm{R}} \leq \frac{1}{2}\left(D_{\mathrm{S}}+2 r\right)$.

\section{B. Proof of Proposition 8}

Proof: For all $\mathbf{x} \in \mathrm{GF}\left(q^{m-1}\right)^{m-1}$ with rank $m-1$, define $g: \mathbf{x} \mapsto \mathbf{y} \in \mathrm{GF}\left(q^{m}\right)^{m}$ such that

$$
\mathbf{Y}=\left(\begin{array}{cc}
\mathbf{X} & \mathbf{0} \\
\mathbf{0} & 1
\end{array}\right) \in \mathrm{GF}(q)^{m \times m},
$$

where $\mathbf{X}$ and $\mathbf{Y}$ are the expansions of $\mathbf{x}$ and $\mathbf{y}$, respectively. By (32), for all $\mathbf{x}, \mathbf{x}^{\prime} \in \mathrm{GF}\left(q^{m-1}\right)^{m-1}$ with rank $m-1$, we have $\operatorname{rk}(g(\mathbf{x}))=\operatorname{rk}(\mathbf{x})+1=m$ and $\operatorname{rk}\left(g(\mathbf{x})-g\left(\mathbf{x}^{\prime}\right)\right)=\operatorname{rk}\left(\mathbf{x}-\mathbf{x}^{\prime}\right)$. Therefore $g$ is a homomorphism from $K_{q}(m-1, m-1, d, m-1)$ to $K_{q}(m, m, d, m)$. Applying (6) to these graphs, and noticing that $\alpha(m, m)=q^{m-1}\left(q^{m}-1\right) \alpha(m-1, m-1)$, we obtain (12). 
We now prove (13). Note that any vector $\mathbf{x} \in \operatorname{GF}\left(q^{m}\right)^{n}$ with rank $r$ belongs to $\left[\begin{array}{c}n-r \\ 1\end{array}\right]$ ELSs of dimension $n-1$. Indeed, such ELSs are those which contain $\mathcal{E}$, where $\mathcal{E}$ is the unique ELS of dimension $r$ such that $\mathbf{x} \in \mathcal{E}$. Using basic counting, there are exactly $\left[\begin{array}{c}n-r \\ 1\end{array}\right]$ such ELSs.

Let $C$ be an optimal $(n, d, r) \operatorname{CRC}$ over $\operatorname{GF}\left(q^{m}\right)$. For all $\mathbf{c} \in C$ and all $\mathcal{V} \in E_{n-1}\left(q^{m}, n\right)$, we define $f(\mathcal{V}, \mathbf{c})=1$ if $\mathbf{c} \in \mathcal{V}$ and $f(\mathcal{V}, \mathbf{c})=0$ otherwise. For all $\mathbf{c}, \sum_{\mathcal{V} \in E_{n-1}\left(q^{m}, n\right)} f(\mathcal{V}, \mathbf{c})=\left[\begin{array}{c}n-r \\ 1\end{array}\right]$, and for all $\mathcal{V}, \sum_{\mathbf{c} \in C} f(\mathcal{V}, \mathbf{c})=|C \cap \mathcal{V}|$. Summing over all possible pairs, we obtain

$$
\begin{aligned}
\sum_{\mathcal{V} \in E_{n-1}\left(q^{m}, n\right)} \sum_{\mathbf{c} \in C} f(\mathcal{V}, \mathbf{c}) & =\sum_{\mathcal{V} \in E_{n-1}\left(q^{m}, n\right)}|C \cap \mathcal{V}| \\
\sum_{\mathbf{c} \in C} \sum_{\mathcal{V} \in E_{n-1}\left(q^{m}, n\right)} f(\mathcal{V}, \mathbf{c}) & =\left[\begin{array}{c}
n-r \\
1
\end{array}\right] A_{\mathrm{R}}\left(q^{m}, n, d, r\right) .
\end{aligned}
$$

Hence there exists $\mathcal{U} \in E_{n-1}\left(q^{m}, n\right)$ such that $|C \cap \mathcal{U}|=\sum_{\mathbf{c} \in C} f(\mathcal{U}, \mathbf{c}) \geq \frac{\left[\begin{array}{c}n-r \\ 1\end{array}\right]}{\left[\begin{array}{c}n \\ 1\end{array}\right]} A_{\mathrm{R}}\left(q^{m}, n, d, r\right)$. The restriction of $C \cap \mathcal{U}$ to the ELS $\mathcal{U}[35]$ is an $(n-1, d, r)$ CRC with cardinality $|C \cap \mathcal{U}|$ over $\operatorname{GF}\left(q^{m}\right)$, and hence $\frac{q^{n-r}-1}{q^{n}-1} A_{\mathrm{R}}\left(q^{m}, n, d, r\right) \leq|C \cap \mathcal{U}| \leq A_{\mathrm{R}}\left(q^{m}, n-1, d, r\right)$.

\section{Proof of Proposition 12}

Proof: For all $\mathbf{x} \in \mathrm{GF}\left(q^{l}\right)^{k}$ with rank $s$ and $\mathbf{c} \in C$, we define $f_{r}(\mathbf{x}, \mathbf{c})=1$ if $d_{\mathrm{R}}(\mathbf{x}, \mathbf{c})=r$ and $f_{r}(\mathbf{x}, \mathbf{c})=0$ otherwise. Note that $\sum_{\mathbf{x}: \mathrm{rk}(\mathbf{x})=s} f_{r}(\mathbf{x}, \mathbf{c})=J\left(q^{l}, k, s, r, \operatorname{rk}(\mathbf{c})\right)$ for all $\mathbf{c} \in C$ and $\sum_{\mathbf{c} \in C} f_{r}(\mathbf{x}, \mathbf{c})=|\{\mathbf{y} \in C-\mathbf{x}: \operatorname{rk}(\mathbf{y})=r\}| \leq A_{\mathrm{R}}\left(q^{l}, k, d, r\right)$ for all $\mathbf{x} \in \mathrm{GF}\left(q^{l}\right)^{k}$. We obtain

$$
\begin{aligned}
& \sum_{\mathbf{c} \in C} \sum_{\mathbf{x}: \mathrm{rk}(\mathbf{x})=s} f_{r}(\mathbf{x}, \mathbf{c})=\sum_{i=0}^{n} A_{i} J\left(q^{l}, k, s, r, i\right), \\
& \sum_{\mathbf{x}: \mathrm{rk}(\mathbf{x})=s} \sum_{\mathbf{c} \in C} f_{r}(\mathbf{x}, \mathbf{c}) \leq N_{s}\left(q^{l}, k\right) A_{\mathrm{R}}\left(q^{l}, k, d, r\right) .
\end{aligned}
$$

Combining (33) and (34), we obtain

$$
A_{\mathrm{R}}\left(q^{m}, n, d, r\right) \geq \frac{\sum_{i=0}^{n} A_{i} J\left(q^{l}, k, s, r, i\right)}{N_{s}\left(q^{l}, k\right)} .
$$

Suppose $d>r+1$. For all $\mathbf{c} \in C$, let us denote the set of vectors with rank $s$ at distance at most $d-r-1$ from $\mathbf{c}$ as $S_{\mathbf{c}}$, and $S \stackrel{\text { def }}{=} \bigcup_{\mathbf{c} \in C} S_{\mathbf{c}}$. For $\mathbf{x} \in S_{\mathbf{c}}$, we have $d_{\mathrm{R}}(\mathbf{x}, \mathbf{c}) \leq d-r-1<r$. We have for $\mathbf{c}^{\prime} \in C$ and $\mathbf{c}^{\prime} \neq \mathbf{c}, d_{\mathrm{R}}\left(\mathbf{x}, \mathbf{c}^{\prime}\right) \geq d_{\mathrm{R}}\left(\mathbf{c}, \mathbf{c}^{\prime}\right)-d_{\mathrm{R}}(\mathbf{x}, \mathbf{c}) \geq r+1$; and hence $f_{r}\left(\mathbf{x}, \mathbf{c}^{\prime}\right)=0$ for all $\mathbf{c}^{\prime} \in C$. Therefore, $\sum_{\mathbf{c} \in C} f_{r}(\mathbf{x}, \mathbf{c})=0$ for all $\mathbf{x} \in S$ and

$$
\sum_{\mathbf{x}: \mathrm{rk}(\mathbf{x})=s} \sum_{\mathbf{c} \in C} f_{r}(\mathbf{x}, \mathbf{c})=\sum_{\mathbf{x} \in S} \sum_{\mathbf{c} \in C} f_{r}(\mathbf{x}, \mathbf{c})+\sum_{\substack{\mathbf{x} \notin S \\ \operatorname{rk}(\mathbf{x})=s}} \sum_{\mathbf{c} \in C} f_{r}(\mathbf{x}, \mathbf{c}) \leq\left[N_{s}\left(q^{l}, k\right)-|S|\right] A_{\mathrm{R}}\left(q^{l}, k, d, r\right) .
$$


Since $d-r-1<\frac{d}{2}$, the balls with radius $d-r-1$ around the codewords are disjoint and hence $|S|=\sum_{i=0}^{n} A_{i} \sum_{t=0}^{d-r-1} J\left(q^{l}, k, s, t, i\right)$. Combining (33) and (36), we obtain

$$
A_{\mathrm{R}}\left(q^{l}, k, d, r\right) \geq \frac{\sum_{i=0}^{n} A_{i} J\left(q^{l}, k, s, r, i\right)}{N_{s}\left(q^{l}, k\right)-\sum_{i=0}^{n} A_{i} \sum_{t=0}^{d-r-1} J\left(q^{l}, k, s, t, i\right)} .
$$

Note that (35) and (37) both hold for any $s$ and weight spectrum $\left\{A_{i}\right\}$. Furthermore, since $A_{\mathrm{R}}\left(q^{l}, k, d, r\right)$ is a non-decreasing function of $l$ and $k, A_{\mathrm{R}}\left(q^{m}, n, d, r\right) \geq A_{\mathrm{R}}\left(q^{l}, k, d, r\right)$ for all $\max \{r, d\} \leq k \leq n$ and $k \leq l \leq m$. Thus, we have (18) and (19).

\section{Proof of Proposition 15}

Proof: By Proposition 11, we obtain $A_{\mathrm{R}}\left(q^{m}, m, d, m\right) \leq \alpha(m, m-d+1)$ for $r=n=m$ and $A_{\mathrm{R}}\left(q^{m}, n, d, r\right) \leq\left[\begin{array}{l}n \\ r\end{array}\right] \alpha(m, r-d+1)<\left[\begin{array}{l}n \\ r\end{array}\right] q^{m(r-d+1)}$ otherwise. We now derive lower bounds on $M\left(q^{m}, n, d, r\right)$. Again, $M\left(q^{m}, n, d, r\right)=\left[\begin{array}{l}n \\ r\end{array}\right] \sum_{j=d}^{r}(-1)^{j} \mu_{j}$ where $\mu_{j}>\mu_{j-1}$ for $d+1 \leq j \leq r$. Therefore, when needed, we shall only consider the last terms in the summation.

First, $M\left(q^{m}, m, m-1, m\right)=\left(q^{2 m}-1\right)-\frac{q^{m}-1}{q-1}\left(q^{m}-1\right)>\frac{q-2}{q-1}\left(q^{2 m}-1\right)>\frac{q-2}{q-1} \alpha(m, 2)$, which leads to (24). For $q=2, M\left(2^{m}, m, m-1, m\right)=2\left(2^{m}-1\right)=\left(2^{m-1}-1\right)^{-1} \alpha(m, 2)$, which results in (23).

Second, when $r=n=m$ and $d=m-2$,

$$
\begin{aligned}
M\left(q^{m}, m, m-2, m\right) & =\left(q^{3 m}-1\right)-\frac{\alpha(m, 1)}{q-1}\left(q^{2 m}-1\right)+\frac{\alpha(m, 2)}{\left(q^{2}-1\right)(q-1)}\left(q^{m}-1\right) \\
& >\frac{q-2}{q-1} \alpha(m, 1)\left(q^{2 m}-1\right)+\frac{1}{\left(q^{2}-1\right)(q-1)} \alpha(m, 2)\left(q^{m}-1\right) \\
& >\frac{\left(q^{2}-1\right)(q-2)+1}{\left(q^{2}-1\right)(q-1)} \alpha(m, 1),
\end{aligned}
$$

which leads to (25).

Third, when $r=n=m$ and $d<m-2$, by considering the last four terms in the summation, we obtain

$$
\begin{aligned}
M\left(q^{m}, m, d, m\right) & >\left(q^{m(m-d+1)}-1\right)-\frac{\alpha(m, 1)}{q-1}\left(q^{m(m-d)}-1\right) \\
& +\frac{\alpha(m, 2)}{\left(q^{2}-1\right)(q-1)}\left(q^{m(m-d-1)}-1\right)-\frac{\alpha(m, 3)}{\left(q^{3}-1\right)\left(q^{2}-1\right)(q-1)}\left(q^{m(m-d-2)}-1\right) \\
& >\left\{\frac{q-2}{q-1}+\frac{q^{3}-2}{\left(q^{3}-1\right)\left(q^{2}-1\right)(q-1)}\right\} \alpha(m, m-d+1),
\end{aligned}
$$

which results in (26). 
Fourth, when $d<r<m$, by considering the last two terms in the summation, we obtain

$$
\begin{aligned}
M\left(q^{m}, n, d, r\right) & \geq\left[\begin{array}{l}
n \\
r
\end{array}\right]\left(\left(q^{m(r-d+1)}-1\right)-\left[\begin{array}{l}
r \\
1
\end{array}\right]\left(q^{m(r-d)}-1\right)\right) \\
& \geq\left[\begin{array}{l}
n \\
r
\end{array}\right]\left(q^{m(r-d+1)}-1-q^{m(r-d)+r}+q^{r}\right) \\
& \geq\left[\begin{array}{l}
n \\
r
\end{array}\right] q^{m(r-d+1)}\left(1-q^{r-m}\right) .
\end{aligned}
$$

Therefore, since $r<m, B\left(q^{m}, n, d, r\right)<\left(1-q^{r-m}\right)^{-1} \leq \frac{q}{q-1}$, which leads to (27).

\section{E. Proof of Proposition 17}

Proof: We first derive a lower bound on $a_{\mathrm{R}}(\nu, \delta, \rho)$. We shall use the following bounds on the Gaussian polynomial: $q^{r(n-r)} \leq\left[\begin{array}{l}n \\ r\end{array}\right]<K_{q}^{-1} q^{r(n-r)}$, where $K_{q} \stackrel{\text { def }}{=} \prod_{j=1}^{\infty}\left(1-q^{-j}\right)$ [35, Lemma 1]. For $d \leq r$, Proposition 14 yields $A_{\mathrm{R}}\left(q^{m}, n, d, r\right) \geq q^{r(n-r)+m(r-d)}$, which asymptotically becomes $a_{\mathrm{R}}(\nu, \delta, \rho) \geq \rho(1+\nu-\rho)-\delta$ for $\delta \leq \rho$. Similarly, for $d>r$, Proposition 16 yields $A_{\mathrm{R}}\left(q^{m}, n, d, r\right) \geq$ $q^{r(n-r)+n(r-d+1)}$, which asymptotically becomes $a_{\mathrm{R}}(\nu, \delta, \rho) \geq \rho(2 \nu-\rho)-\nu \delta$ for $\delta \geq \rho$. Also, by (5), (9) asymptotically yields $a_{\mathrm{R}}(\nu, \delta, \rho) \geq \min \left\{(\nu-\rho)\left(\rho-\frac{\delta}{2}\right), \rho\left(\nu-\rho-\frac{\delta}{2}\right)\right\}$ for $\delta \geq \rho$.

Proposition 5 and (5) yield $\log _{q} A_{\mathrm{R}}\left(q^{m}, n, d, r\right) \geq \min \{(n-r)(2 r-d-2 p+1),(m-r)(p+1)\}$ for $d>r$ and $2 r \leq n$. Treating the two terms as functions and assuming that $p$ is real, the lower bound is maximized when

$$
p=\frac{(n-r)(2 r-d+1)-m+r}{m+2 n-3 r} .
$$

Using $p=\left\lfloor\frac{(n-r)(2 r-d+1)-m+r}{m+2 n-3 r}\right\rfloor$, asymptotically we obtain $a_{\mathrm{R}}(\nu, \delta, \rho) \geq \frac{(1-\rho)(\nu-\rho)}{1+2 \nu-3 \rho}(2 \rho-\delta)$ for $2 \rho \leq \nu$.

For $d>r$ and $n \leq 2 r \leq m$, Proposition 5 and (5) lead to $\log _{q} A_{\mathrm{R}}\left(q^{m}, n, d, r\right) \geq \min \{r(n-d-2 p+$ $1),(m-r)(p+1)\}$. After maximizing this expression over $p$, we asymptotically obtain $a_{\mathrm{R}}(\nu, \delta, \rho) \geq$ $\frac{\rho(1-\rho)}{1+\rho}(\nu-\delta)$ for $\nu \leq 2 \rho \leq 1$.

For $d>r$ and $2 r \geq m$, Proposition [5 and (5) lead to $\log _{q} A_{\mathrm{R}}\left(q^{m}, n, d, r\right) \geq \min \{r(n-d-2 p+$ $1), r(m-2 r+p+1)\}$. After maximizing this expression over $p$, we asymptotically obtain $a_{\mathrm{R}}(\nu, \delta, \rho) \geq$ $\frac{\rho}{3}(2-4 \rho+\nu-\delta)$ for $2 \rho \geq 1$.

We now derive an upper bound on $a_{\mathrm{R}}(\nu, \delta, \rho)$. First, Proposition 11 gives $A_{\mathrm{R}}\left(q^{m}, n, d, r\right)<\left[\begin{array}{l}n \\ r\end{array}\right] q^{m(r-d+1)}<$ $K_{q}^{-1} q^{r(n-r)+m(r-d+1)}$ for $d \leq r$, which asymptotically becomes $a_{\mathrm{R}}(\nu, \delta, \rho) \leq \rho(1+\nu-\rho)-\delta$ for $\rho \geq \delta$. Second, by Proposition [5, we obtain $a_{\mathrm{R}}(\nu, \delta, \rho) \leq \lim _{m \rightarrow \infty} \sup \left[\log _{q^{m^{2}}} A_{\mathrm{S}}(q, n, 2(d-r), r)\right]=$ $\min \{(\nu-\rho)(2 \rho-\delta), \rho(\nu-\delta)\}$ for $\rho \leq \delta \leq \min \{2 \rho, \nu\}$. 


\section{REFERENCES}

[1] T. Ho, R. Koetter, M. Médard, D. R. Karger, and M. Effros, "The benefits of coding over routing in a randomized setting," in Proc. IEEE Int. Symp. Information Theory, Yokohama, June-July 2003, p. 442.

[2] P. A. Chou, Y. Wu, and K. Jain, "Practical network coding," in Allerton Conf. on Comm., Control, and Computing, Monticello, IL, October 2003.

[3] T. Ho, M. Médard, R. Koetter, D. R. Karger, M. Effros, J. Shi, and B. Leong, "A random linear network coding approach to multicast," IEEE Trans. Info. Theory, vol. 52, no. 10, pp. 4413-4430, October 2006.

[4] N. Cai and R. W. Yeung, "Network coding and error correction," in Proc. IEEE Info. Theory Workshop, Bangalore, India, October 2002, pp. 20-25.

[5] L. Song, R. W. Yeung, and N. Cai, “Zero-error network coding for acyclic networks," IEEE Trans. Info. Theory, vol. 49, no. 12, pp. 3129-3139, December 2003.

[6] R. W. Yeung and N. Cai, "Network error correction, part I: Basic concepts and upper bounds," Commun. Inform. Syst., vol. 6, no. 1, pp. 19-36, 2006.

[7] N. Cai and R. W. Yeung, "Network error correction, part II: Lower bounds," Commun. Inform. Syst., vol. 6, no. 1, pp. 37-54, 2006.

[8] Z. Zhang, "Linear network error correction codes in packet networks," IEEE Trans. Info. Theory, vol. 54, no. 1, pp. 209-218, January 2008.

[9] R. Koetter and F. R. Kschischang, "Coding for errors and erasures in random network coding," IEEE Trans. Info. Theory, vol. 54, no. 8, pp. 3579-3591, August 2008.

[10] D. Silva, F. R. Kschischang, and R. Koetter, "A rank-metric approach to error control in random network coding," submitted to IEEE Trans. Info. Theory, available at http://arxiv.org/abs/0711.0708

[11] S. Jaggi, M. Langberg, S. Katti, T. Ho, D. Katabi, and M. Médard, "Resilient network coding in the presence of Byzantine adversaries," in Proc. IEEE Infocom, Anchorage, AK, May 2007, pp. 616-624.

[12] P. Delsarte, "Association schemes and $t$-designs in regular semilattices," Journal of Combinatorial Theory A, vol. 20, pp. 230-243, 1976.

[13] L. Chihara, "On the zeros of the Askey-Wilson polynomials with applications to coding theory," SIAM J. Math. Anal., vol. 8, no. 1, pp. 191-207, January 1987.

[14] T. Etzion and A. Vardy, "Error-correcting codes in projective space," in Proc. IEEE Int. Symp. Info. Theory, Toronto, ON, July 2008, pp. 871-875.

[15] E. M. Gabidulin and M. Bossert, "Codes for network coding," in Proc. IEEE Int. Symp. Info. Theory, Toronto, ON, July 2008, pp. 867-870.

[16] W. J. Martin and X. J. Zhu, "Anticodes for the Grassmann and bilinear forms graphs," Designs, Codes and Cryptography, vol. 6, pp. 73-79, 1995.

[17] R. Ahlswede, H. K. Aydinian, and L. H. Khachatrian, "On perfect codes and related concepts," Designs, Codes and Cryptography, vol. 22, pp. 221-237, 2001.

[18] M. Schwartz and T. Etzion, “Codes and anticodes in the Grassmann graph,” J. Combin. Theory Ser. A, vol. 97, pp. 27-42, 2002.

[19] S.-T. Xia and F.-W. Fu, "Johnson type bounds on constant dimension codes," submitted to Designs, Codes and Cryptography, Sept. 2007, available at http://arxiv.org/abs/0709.1074 
[20] P. Frankl and R. M. Wilson, “The Erdös-Ko-Rado theorem for vector spaces,” Journal of Combinatorial Theory A, vol. 43, pp. 228-236, 1986.

[21] T. Etzion, "Perfect byte-correcting codes," IEEE Trans. Info. Theory, vol. 44, pp. 3140-3146, 1998.

[22] H. Wang, C. Xing, and R. Safani-Naini, "Linear authentication codes: Bounds and constructions," IEEE Trans. Info. Theory, vol. 49, no. 4, pp. 866-872, April 2003.

[23] V. Skachek, "Recursive code construction for random networks," 2008, available at http://arxiv.org/abs/0806.3650v1

[24] D. Silva and F. R. Kschischang, "On metrics for error correction in network coding," 2008, available at http://arxiv.org/abs/0805.3824v1

[25] P. Delsarte, "Bilinear forms over a finite field, with applications to coding theory," Journal of Combinatorial Theory A, vol. 25 , pp. 226-241, 1978.

[26] E. M. Gabidulin, "Theory of codes with maximum rank distance," Problems on Information Transmission, vol. 21, no. 1, pp. 1-12, Jan. 1985.

[27] R. M. Roth, "Maximum-rank array codes and their application to crisscross error correction," IEEE Trans. Info. Theory, vol. 37, no. 2, pp. 328-336, March 1991.

[28] E. M. Gabidulin, A. V. Paramonov, and O. V. Tretjakov, "Ideals over a non-commutative ring and their application in cryptology," LNCS, vol. 573, pp. 482-489, 1991.

[29] P. Lusina, E. M. Gabidulin, and M. Bossert, "Maximum rank distance codes as space-time codes," IEEE Trans. Info. Theory, vol. 49, pp. 2757-2760, Oct. 2003.

[30] A. Kshevetskiy and E. M. Gabidulin, "The new construction of rank codes," Proc. IEEE Int. Symp. on Information Theory, pp. 2105-2108, Sept. 2005.

[31] G. E. Andrews, The Theory of Partitions, ser. Encyclopedia of Mathematics and its Applications, G.-C. Rota, Ed. Reading, MA: Addison-Wesley, 1976, vol. 2.

[32] E. Bannai and T. Ito, Algebraic Cominatorics I. Association Schemes. The Benjamin/Cummings Publishing Company, 1983.

[33] P. Delsarte, "Properties and applications of the recurrence $F(i+1, k+1, n+1)=q^{k+1} F(i, k+1, n)-q^{k} F(i, k, n)$," SIAM Journal of Applied Mathematics, vol. 31, no. 2, pp. 262-270, September 1976.

[34] M. Gadouleau and Z. Yan, "MacWilliams identity for codes with the rank metric," EURASIP Journal on Wireless Communications and Networking, vol. 2008, Special issue: Advances in Error Control Coding Techniques, 2008.

[35] — - "On the decoder error probability of bounded rank-distance decoders for maximum rank distance codes," IEEE Trans. Info. Theory, vol. 54, no. 7, pp. 3202-3206, July 2008.

[36] S. Y. El Rouayheb, C. N. Georghiades, E. Soljanin, and A. Sprintson, "Bounds on codes based on graph theory," Proc. IEEE Int. Symp. on Information Theory, pp. 1876-1879, June 2007.

[37] C. D. Godsil and G. Royle, Algebraic Graph Theory, ser. Graduate Texts in Mathematics. Springer-Verlag, 2001 , vol. 207.

[38] A. R. Rao and P. Bhimasankaram, Linear Algebra, 2nd ed. Hindustan Book Agency, May 2000.

[39] M. Gadouleau and Z. Yan, "Packing and covering properties of rank metric codes," to appear in IEEE Trans. Info. Theory, available at http://arxiv.org/abs/cs/0701098

[40] S. M. Johnson, "A new upper bound for error-correcting codes," IRE Trans. Info. Theory, vol. 8, pp. $203-207,1962$.

[41] L. A. Bassalygo, "New upper bounds for error correcting codes," Problems of Information Transmission, vol. 1, no. 4, pp. 32-35, 1968. 
[42] M. Gadouleau and Z. Yan, "Properties of codes with the rank metric," in Proc. IEEE Globecom, San Francisco, CA, November 2006, pp. 1-5. 